(W)

Check for

updates

Cite as

Nano-Micro Lett.

(2019) 11:74

Received: 5 July 2019

Accepted: 18 August 2019

Published online: 9 September 2019

(C) The Author(s) 2019

\title{
Inorganic Nanozyme with Combined Self-Oxygenation/Degradable Capabilities for Sensitized Cancer Immunochemotherapy
}

Jie Wang ${ }^{1}$, Lan Fang ${ }^{1}$, Ping $\mathrm{Li}^{1}$, Lang $\mathrm{Ma}^{2,3}$, Weidan $\mathrm{Na}^{1}$, Chong Cheng ${ }^{2,3} \bowtie$, Yueqing $\mathrm{Gu}^{1}$, Dawei Deng ${ }^{1,4} \bowtie$

$\triangle$ Chong Cheng, chong.cheng@fu-berlin.de; Dawei Deng, dengdawei@cpu.edu.cn

1 Department of Pharmaceutical Engineering and Department of Biomedical Engineering, School of Engineering, China Pharmaceutical University, Nanjing 211198, People's Republic of China

2 Institute of Chemistry and Biochemistry, Freie Universität Berlin, Takustrasse 3, 14195 Berlin, Germany

3 State Key Laboratory of Polymer Materials Engineering, College of Polymer Science and Engineering, Sichuan University, Chengdu 610065, People's Republic of China

4 State Key Laboratory of Natural Medicines, National R\&D Center for Chinese Herbal Medicine Processing, China Pharmaceutical University, Nanjing 211198, People's Republic of China

\section{HIGHLIGHTS}

- Self-oxygenation/degradable nanozyme reactors with core-shell structure were fabricated for relieving tumor hypoxia.

- Intratumoral hypoxia alleviation and sensitization of immunochemotherapy using as-prepared nanozyme reactors was demonstrated in B16F10 melanoma tumor.

\begin{abstract}
Recently emerged cancer immunochemotherapy has provided enormous new possibilities to replace traditional chemotherapy in fighting tumor. However, the treatment efficacy is hampered by tumor hypoxiainduced immunosuppression in tumor microenvironment (TME). Herein, we fabricated a self-oxygenation/degradable inorganic nanozyme with a core-shell structure to relieve tumor hypoxia in cancer immunochemotherapy. By integrating the biocompatible $\mathrm{CaO}_{2}$ as the oxygen-storing component, this strategy is more effective than the earlier designed nanocarriers for delivering oxygen or $\mathrm{H}_{2} \mathrm{O}_{2}$, and thus provides remarkable oxygenation and long-term capability in relieving hypoxia throughout the tumor tissue. Consequently, in vivo tests validate that the delivery system can successfully relieve hypoxia and reverse the immunosuppressive TME to favor antitumor immune responses, leading to enhanced chemoimmunotherapy with cytotoxic $\mathrm{T}$ lymphocyte-associated antigen 4 blockade. Overall, a facile, robust and effective strategy is proposed to improve tumor oxygenation by using self-decomposable and biocompatible inorganic nanozyme reactor, which will not only provide an innovative pathway to relieve

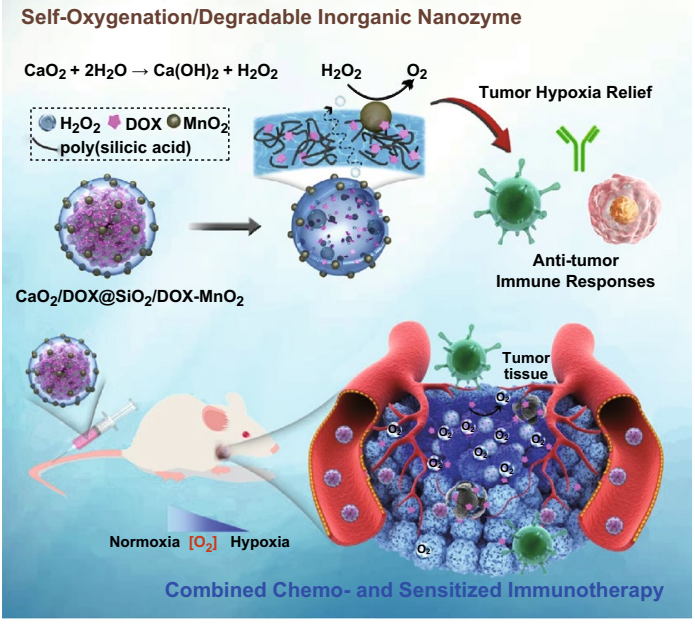
intratumoral hypoxia, but also present potential applications in other oxygen-favored cancer therapies or oxygen deficiency-originated diseases.
\end{abstract}

KEYWORDS Inorganic nanozyme; Self-oxygenation nanoreactor; Biodegradable nanomedicine; Immunochemotherapy; Cancer treatment 


\section{Introduction}

Cancer immunochemotherapy that integrates chemotherapeutic and immune-modulating agents has attracted increasing attentions due to the synergistically enhanced antitumor effects [1]. However, the development of immunochemotherapy is hampered by the lack of a nanoplatform that can effectively and simultaneously achieve both therapeutic goals in tumor tissues [2]. In the emerging immunochemotherapy for cancer treatment, it is often encountered that the immune system fails to respond to the tumor cells [3], even when a large number of antitumor $\mathrm{T}$ cells are present [4]. It has been clear that the TME, featured with low $\mathrm{pH}$ values, insufficient blood flow, overproduced peroxide and hypoxia, plays a vital role in such failures for tumor immunosuppression [5-7]. Among these characteristics, the hypoxia has been reported as one of the most unfavorable features that tend to suppress the immunotherapeutic efficiency [8-10]. In many in vivo situations, the activation and effector function of antitumor T cells can be diminished in the hypoxic TME, which thus protect tumor cells from being eliminated via immune system [11]. A dominant pathway in such immunosuppression is the hypoxia-adenosinergic signaling [12], because of which the immune-cell function will be negatively regulated, the production of effector cytokines such as interferon- $\gamma(\mathrm{IFN}-\gamma)$ will be reduced and inhibitors of this signaling such as A2AR antagonist and extracellular adenosine-degrading drugs have been used as adjuvant to compromise the hypoxia-induced tumor protection. Hence, modulating the unfavorable hypoxia characteristic of TME has been considered as an effective and clinically valuable strategy for improving tumor immunotherapy [13, 14].

To overcome the tumor hypoxia-induced immunosuppression, different strategies have been proposed recently to enhance the tumor oxygenation, for instance by increasing the intratumoral blood flow via normalization of tumor vasculatures [15], or using perfluorocarbon to deliver oxygen to hypoxic tumor tissue [16]. A direct and robust approach proposed by Sitkovsky's group was to provide supplementary oxygen, in which tumor-bearing mice were placed in chambers containing $60 \%$ oxygen for respiratory hyperoxia [17-19]. This treatment effectively weakened hypoxia and alleviated immunosuppression, resulting in strong tumor rejection $[18,19]$. Meanwhile, it has been proved that the tumor cells can produce excessive amounts of $\mathrm{H}_{2} \mathrm{O}_{2}$ within the TME by the overexpressed superoxide dismutase [20], which thus has been utilized for producing $\mathrm{O}_{2}$ to relieve the tumor hypoxia [21]. The development of nanotechnology offers new opportunities to address the shortcomings of conventional designs [22-28]; inorganic nanomaterials with catalase mimetic properties, such as the $\mathrm{MnO}_{2}$ and carbon nitride-based nanostructures, have been designed to generate oxygen in tumor tissue [29-35]. However, the concentration of endogenous $\mathrm{H}_{2} \mathrm{O}_{2}$ is extremely low, generally reported to be $50-100 \times 10^{-6} \mathrm{M},[20,36]$ which is too low to generate sufficient amount of oxygen for inducing satisfactory tumor hypoxia relief [20]. Very recently, the tumor oxygenation by delivering exogenous $\mathrm{H}_{2} \mathrm{O}_{2}$ and the subsequent catalase-triggered $\mathrm{H}_{2} \mathrm{O}_{2}$ decomposition/oxygen generation has been proposed to offer a more effective approach for tumor oxygenation [37]. However, direct delivery of $\mathrm{H}_{2} \mathrm{O}_{2}$ via liposomes or $\mathrm{H}_{2} \mathrm{O}_{2}$-related particles possess several intrinsic drawbacks, such as difficulty in preparation and storage, relatively low stability after injection, and hard to control the releasing speed of $\mathrm{H}_{2} \mathrm{O}_{2}$ and maintain the activity of catalase, which will undoubtedly hamper its practical application for tumor hypoxia. Therefore, fabricating more robust and facile alternative with enhanced physiological stability and lowered cost is highly desirable and necessary.

Herein, we report a self-oxygenation/degradable inorganic nanozyme reactor (NR) that can effectively relieve tumor hypoxia for sensitized cancer immunochemotherapy. The core of nanozyme reactor is composed of $\mathrm{CaO}_{2}$ /doxorubicin (DOX), and the shell consists of degradable $\mathrm{SiO}_{2} / \mathrm{DOX}-$ $\mathrm{MnO}_{2}$; thus, this whole nanoplatform is named as $\mathrm{CaO}_{2} /$ DOX @ $\mathrm{SiO}_{2} / \mathrm{DOX}-\mathrm{MnO}_{2}(\mathrm{CDSDM}) \mathrm{NR} . \mathrm{CaO}_{2}$ can be gradually hydrolyzed to generate $\mathrm{H}_{2} \mathrm{O}_{2}$, which as the "fuel" can diffuse through the shell and decompose in the presence of $\mathrm{MnO}_{2}$ to produce oxygen. Meanwhile, the embedded DOX in $\mathrm{CaO}_{2}$ core and $\mathrm{SiO}_{2}$ shell allows efficient chemotherapy and also facilitates the self-decomposition of the resulting $\mathrm{SiO}_{2} / \mathrm{DOX}$ structure. Compared with nanoparticles without $\mathrm{CaO}_{2}$ core that can only decompose the tumor endogenous $\mathrm{H}_{2} \mathrm{O}_{2}$, the $\mathrm{CaO}_{2}$ core-contained CDSDM NR provides much remarkable oxygenation and long-term capability in relieving hypoxia throughout the tumor tissue.

In vivo tests also validate that treatment with these CDSDM NRs can efficiently relieve hypoxia in B16F10 melanoma tumor model, thus reversing the immunosuppressive TME to favor antitumor immunities. By combining cytotoxic $\mathrm{T}$ lymphocyte-associated antigen 4 (CTLA-4) 
blockade-mediated immunotherapy with CDSDM NR treatment, strong tumor regression was observed. Further studies reveal that CDSDM NR treatment can enhance the intratumoral infiltration of antitumor $\mathrm{CD}^{+} \mathrm{T}$ cells and decrease the population of immunosuppressive regulatory $\mathrm{T}$ cells (Tregs). Moreover, it has been noticed that these CDSDM NRs show no obvious toxicity. Therefore, our TME-motivated self-oxygenation/degradable inorganic nanozyme reactor not only provides an innovative and facile tumor oxygenation strategy to relieve tumor hypoxia, but also holds great potential in other oxygen-favored therapies and oxygen deficiency-originated diseases, as well as in anti-pathogen treatments [38].

\section{Experimental}

\subsection{Synthesis of CSDM and CDSDM NRs}

Preparation of $\mathrm{CaO}_{2}$ and $\mathrm{CaO}_{2} / \mathrm{DOX}$ cores: The method was derived from the gas diffusion-controlled preparation of $\mathrm{CaCO}_{3}$ NPs. Typically, $50 \mathrm{mg}$ of $\mathrm{CaCl}_{2} \cdot 2 \mathrm{H}_{2} \mathrm{O}$ was dissolved in $50 \mathrm{~mL}$ of ethanol in a beaker, into which $0.2 \mathrm{~mL}$ of $30 \% \mathrm{H}_{2} \mathrm{O}_{2}$ solution was added. The beaker was then covered by parafilm with a few holes and left in a desiccator at $35{ }^{\circ} \mathrm{C}$ together with a glass bottle containing $2 \mathrm{~mL}$ of ammonia. After $2 \mathrm{~h}$ of reaction, white colloid was obtained in the beaker. The product was centrifuged and washed with ethanol for further use. For preparation of $\mathrm{CaO}_{2} / \mathrm{DOX}$ cores, $5 \mathrm{mg}$ of doxorubicin hydrochloride (DOX $\cdot \mathrm{HCl}, 98 \%$ ) was added into the solution containing $\mathrm{CaCl}_{2}$ and $\mathrm{H}_{2} \mathrm{O}_{2}$ before incubating with ammonia.

Encapsulation of self-decomposable $\mathrm{SiO}_{2} / \mathrm{DOX}$ shell: $4 \mathrm{~mL}$ of about $2.5 \mathrm{mg} \mathrm{mL}^{-1}$ of $\mathrm{CaO}_{2}$ core or $\mathrm{CaO}_{2} / \mathrm{DOX}$ solution was dissolved into $50 \mathrm{~mL}$ absolute ethanol, and then $15 \mathrm{mg}$ of polyvinyl pyrrolidone (PVP, MW 58,000, k29-32) was added. Under stirring, $30 \mu \mathrm{L}$ of $2 \mathrm{M} \mathrm{NaOH}$ solution and $15 \mathrm{mg}$ of $\mathrm{DOX} \cdot \mathrm{HCl}$ are sequentially added. $0.1 \mathrm{~mL}$ of tetraethyl orthosilicate (TEOS, 99.99\%) was then added. After $24 \mathrm{~h}$ of reaction in dark, the solution was centrifuged at $8000 \mathrm{rpm}$ and washed with water three times and dispersed in water for further use.

Preparation and adsorption of CSDM and CDSDM NRs: Ultra-small $\mathrm{MnO}_{2}$ nanodots were first prepared according to previous reports with certain modification [30]. Briefly, $70 \mathrm{mg}$ of $\mathrm{KMnO}_{4}(\geq 99.5 \%)$ and $55 \mathrm{mg}$ of poly(allylamine hydrochloride) (PAH, MW 15,000, 98\%) were separately dissolved into $10 \mathrm{~mL}$ of deionized water. Under stirring, the $\mathrm{KMnO}_{4}$ solution was added dropwise into the PAH solution. After $15 \mathrm{~min}$ of reaction, $0.75 \mathrm{~mL}$ of $\mathrm{pH} 6.5$ phosphate buffer saline (PBS) was added to $10 \mathrm{~mL}$ of the brown solution to disturb the stabilization by PAH. The obtained mixture was centrifuged at $10,000 \mathrm{rpm}$ for $5 \mathrm{~min}$ and washed with water. $5 \mathrm{~mL}$ of about $1 \mathrm{mg} \mathrm{mL}^{-1}$ of $\mathrm{SiO}_{2} / \mathrm{DOX}$ shellencapsulated NP solution was mixed with $0.2 \mathrm{~mL}$ of about $0.5 \mathrm{mg} \mathrm{mL}^{-1} \mathrm{MnO}_{2}$ nanodot solution under stirring, and CDSDM or CSDM NRs were obtained via charge attraction.

\subsection{Characterizations}

UV-Vis spectrophotometer (Shimadzu UV-2550) and fluorescence spectrophotometer (Edinburgh FS5) were used to obtain absorption and photoluminescence (PL) spectra, respectively. Transmission electron microscope (TEM) images were taken using a HT7700 (Hitachi) transmission electron microscope. Selected area diffraction and elemental mapping were acquired using a JEM 2100 (JEOL) transmission electron microscope. X-ray photoelectron spectrometry (XPS) assay was performed using a PHI 5000 VersaProbe (UIVAC-PHI, Japan) spectrometer.

\subsection{Oxygen Generation and DOX Release}

Oxygen generation by NRs was detected based on the decrease in fluorescence intensity of tris(4,7-diphenyl1,10-phenanthroline) ruthenium dichloride $\left(\mathrm{Ru}(\mathrm{dpp})_{3} \mathrm{Cl}_{2}\right.$, $98 \%$ ) in the presence of oxygen. Under $\mathrm{N}_{2}$ atmosphere, CDSDM NRs $\left(\sim 2 \mathrm{mg} \mathrm{mL}^{-1}\right)$ were mixed with ethanolic solution of $\mathrm{Ru}(\mathrm{dpp})_{3} \mathrm{Cl}_{2}(40 \mu \mathrm{M})$ and incubated for different durations. At scheduled time points, the solution was centrifuged ( $8000 \mathrm{rpm}, 3 \mathrm{~min}$ ) and the supernatant was subjected to fluorescence detection with the tube sealed. CDSDM NR solution without $\mathrm{Ru}(\mathrm{dpp})_{3} \mathrm{Cl}_{2}$ was independently tested to exclude the influence of DOX fluorescence. DOX release from CDSDM NRs was measured by dialyzing with filter of 1000 Da cut-off. For in vitro hypoxia level evaluation, B16F10 cells were cultured for $4 \mathrm{~h}$ in $0.5 \mathrm{~L}$ rectangular jar sealed with a pouch, and then $0.2 \mathrm{~mL}$ of PBS, hDSDM $\left(1.2 \mathrm{mg} \mathrm{mL}^{-1}\right)$ or CDSDM $\left(2.5 \mathrm{mg} \mathrm{mL}^{-1}\right) \mathrm{NRs}$ was added, followed by pimonidazole. After $4 \mathrm{~h}$ of incubation, cells were washed and FITC-conjugated anti-pimonidazole (Hypoxyprobe) was added and further co-incubated for $1 \mathrm{~h}$. 
Cell imaging was performed with a laser confocal scanning microscope (LCSM, Olympus Fluoview 1000).

\subsection{Evaluation of In Vivo NR Distribution}

In vivo imaging and histological section imaging were performed to study the in vivo NR distribution. For in vivo imaging, CDSDM NRs were intravenously injected into B16F10 tumor-bearing mice. IVIS In Vivo Imaging System (PerkinElmer) was used to monitor the NR distribution in a mouse based on the fluorescence of DOX $\left(\lambda_{\mathrm{ex}} / \lambda_{\mathrm{ex}}=500 / 610 \mathrm{~nm}\right)$. After different time post-injection, mice were sacrificed and major organs and tumor were collected for imaging and then sliced into sections for histological imaging using LCSM. All in vivo experiments involving mice were approved by the Jiangsu Association for Laboratory Animals and the Department of Science and Technology of Jiangsu Province.

\subsection{Evaluation of Intratumoral Hypoxia}

Balb/c mice with established B16F10 tumors were intravenously injected with saline, hDSDM NPs $(0.2 \mathrm{~mL}$ of $\left.1.2 \mathrm{mg} \mathrm{mL}^{-1}\right)$ or CDSDM NRs $\left(0.2 \mathrm{~mL}\right.$ of $\left.2.5 \mathrm{mg} \mathrm{mL}^{-1}\right)$. Hypoxyprobe-1 Green kit was used to detect hypoxia. Eighthour post-injection, pimonidazole ( $2 \mathrm{mg}$ per mouse) was intravenously injected. $1 \mathrm{~h}$ later, tumors were dissected and cryo-sectioned, which were then co-incubated with anti-pimonidazole antibody. Cell nuclei were stained using 4',6-diamidino-2-phenylindole (DAPI). Photoacoustic imaging and 3D cell culture model were also employed for the evaluation of hypoxia relief. Endra Nexus 128 PA imaging system was used for photoacoustic imaging, which was based on the different peak absorptions of oxyhemoglobin $(\sim 850 \mathrm{~nm})$ and deoxyhemoglobin $(\sim 760 \mathrm{~nm})$. Mice were kept anaesthetized so that the same section of each tumor tissue (about $3 \mathrm{~mm}$ from tumor surface) could be imaged for better comparison. Imaging was performed at 760 and $850 \mathrm{~nm}$ at each time point within $2 \mathrm{~h}$. Three-dimensional tumor spheroids (400-500 $\mu \mathrm{m}$, from KeyGen Co. Ltd., Nanjing) were co-incubated with different agents for $6 \mathrm{~h}$, and pimonidazole hydrochloride was then added for another two of co-incubation. Then spheroids were cryo-sectioned and sequentially stained with anti-pimonidazole antibody and DAPI.

\subsection{Detection of Extracellular Adenosine and Cytokines}

Tumor tissues excised after different treatments were firstly digested into single-cell suspensions. Erythro-9-(2-hydroxy3-nonyl) adenine was added into the filtrates to prevent the degradation of adenosine. The resulting mixture was then centrifuged at $10,000 \mathrm{rpm}$ for $10 \mathrm{~min}$. The supernatants were then filtered with a 3000 Da cut-off filter, and the filtrate was freeze-dried and re-dissolved by water of 1/100 of initial volume. Adenosine measurement was carried out using high-performance liquid chromatography (Agilent 1260) (mobile phases: methanol and $10 \mathrm{mM} \mathrm{KH}_{2} \mathrm{PO}_{4}$, v/v of 1/9). For cytokine detection, serum samples were obtained from mice after above treatments, and mouse interferon $\gamma$ (IFN$\gamma$, Elabscience) and mouse tumor necrosis factor $\alpha$ (TNF- $\alpha$, Elabscience) Elisa kits were used.

\subsection{Antitumor Treatment with NRs and CTLA-4 Blockade}

Mice with established B16F10 tumors $\left(\sim 60 \mathrm{~mm}^{3}\right)$ were randomly divided into five groups. The closely monitored treatment period is 20 days, during which mice were treated with saline, or saline and CTLA-4 blockade, or CSDM NRs, or CDSDM NRs, or hDSDM NPs and CTLA-4 blockade, or CDSDM NRs and CTLA-4 blockade, or CDSDM NRs + CSDM NRs (adaptive administration) and CTLA-4 blockade. In adaptive administration, CDSDM NRs were given firstly, which was replaced by CSDM NRs if tumor size reduced by $10 \%$ or more compared to the last measurement; CDSDM NRs would not be reused until tumor size increased at least $10 \%$ compared to the last measurement. Tumor size was calculated based on the formula: width $^{2} \times$ length/2. Saline, NPs $\left(0.2 \mathrm{~mL}\right.$ of $\left.1.2 \mathrm{mg} \mathrm{mL}^{-1}\right)$ and NRs $\left(0.2 \mathrm{~mL}\right.$ of $\left.2.5 \mathrm{mg} \mathrm{mL}^{-1}\right)$ were given every 2 days, while CTLA-4 blockade $(20 \mu \mathrm{L})$ was given every 4 days. During the treatment, the tumor volume, body weight and body condition (mobility, food taking, palpable infection, etc.) were closely monitored.

\subsection{Western Blot}

Mice with established B16F10 tumor were administrated with saline, hDSDM NRs $\left(0.2 \mathrm{~mL}\right.$ of $\left.1.2 \mathrm{mg} \mathrm{mL}^{-1}\right)$ or CDSDM NRs $\left(0.2 \mathrm{~mL}\right.$ of $\left.2.5 \mathrm{mg} \mathrm{mL}^{-1}\right) .24 \mathrm{~h}$ later, the 
tumors were excised and digested, and the single-cell suspensions were put through a $70 \mu \mathrm{m}$ strainer. Cells were further digested in lysis buffer containing proteinase inhibitor. Lysates were centrifuged at $10,000 \mathrm{rpm}$ for $5 \mathrm{~min}$ at $4{ }^{\circ} \mathrm{C}$ to collect the supernatant. Protein concentration was analyzed via BCA protein assay reagent. After separation with $12 \%$ SDS-PAGE, the proteins were transferred to nitrocellulose membrane. After further incubation with 5\% nonfat milk powder solution, membrane was incubated with primary antibodies including anti-mouse CD39 and anti-mouse CD73 (Santa Cruz) overnight, and horseradish peroxidaseconjugated secondary antibodies were then incubated for $1 \mathrm{~h}$. Enhanced chemiluminescence reagent was used to visualize the bands.

\subsection{Immunofluorescence Staining}

Tumor tissues excised after 4 days of the indicated treatments in main text were embedded by OCT compound and sectioned into $10 \mu \mathrm{m}$ slices. Shallow tissue was defined as about $0-1.5 \mathrm{~mm}$ from surface, while deep tissue was $>2 \mathrm{~mm}$ from surface. (The average diameter of tumor was about 4-6 mm.) After fixed with acetone/methanol (1/1 in v:v), FITC-conjugated anti-HIF- $\alpha$ and Alexa680-conjugated antiCD8 antibodies (Santa Cruz) at a concentration of 1:100 were used for staining. Excitation of CD8 antibodies was carefully filtered to avoid the interference of DOX fluorescence. DAPI was used for staining nuclei.

\subsection{Flow Cytometry}

For analysis of HIF-1 $\alpha$ expression, tumor tissues were excised from mice after different treatments and homogenized with Collagenase D in 1640 cell culture media at $37^{\circ} \mathrm{C}$. The obtained single-cell suspensions were washed and fixed with Fix/Perm, and anti-HIF-1 $\alpha$-FITC (Santa Cruz) was co-incubated for 20 min in dark. For the analysis of intratumoral Tregs, the single-cell suspensions from different groups were filtered with $70 \mu \mathrm{m}$ strainer and washed, and anti-CD25-Alexa Fluor 790 (Santa Cruz), anti-CD4FITC (Santa Cruz) and anti-Foxp3-Alexa Fluor 680 (Santa Cruz) were used for surface staining in dark. For analysis of the maturation of DCs, two peripheral lymph nodes under the armpit at tumor side were isolated, and the obtained single-cell suspensions were stained with anti-CD80-FITC and anti-CD60-Alexa Fluor 680 (Santa Cruz) in dark. A BD FACSCalibur flow cytometer was used in flow cytometry.

\subsection{Statistics}

SPSS Statistics 19 was used for data analysis including standard deviation and significance test. Student's $t$ test was used for the comparison of volume or concentration statistics between the test group and each of other groups.

\section{Results and Discussion}

\subsection{Synthesis and Properties of Oxygen-Self-Produced CDSDM NRs}

The synthetic procedure of CDSDM NRs is illustrated in Fig. 1a; the core of NR is composed of self-decomposable $\mathrm{CaO}_{2} / \mathrm{DOX}$, and the shell consists of biodegradable $\mathrm{SiO}_{2} /$ DOX- $\mathrm{MnO}_{2}$. DOX was incorporated into silica shells to enable the shell self-decomposition, and was further embedded into $\mathrm{CaO}_{2}$ cores to improve the DOX loading capacity. Particularly, the $\mathrm{CaO}_{2}$ cores were prepared via a gas diffusion method (Fig. S1) and showed monodispersed spherical morphology (Fig. S2). High-resolution transmission electron microscope (HRTEM) and selected area electron diffraction (SAED) results demonstrated the $\mathrm{CaO}_{2}$ characteristic crystalline. DOX could be readily incorporated into $\mathrm{CaO}_{2} \mathrm{NPs}$ due to the formation of Ca-DOX complex, despite the more amorphous crystalline of the resulting produces. Unlike the previously reported $\mathrm{H}_{2} \mathrm{O}_{2}$-filled polymersome used for reactive oxygen species-mediated therapy [39], here, the generation of $\mathrm{H}_{2} \mathrm{O}_{2}$ (hydrolysis of $\mathrm{CaO}_{2}$ ) was sustained and preferred in the acidic TME ( $\mathrm{pH} \sim 6.5)$ and endosome ( $\mathrm{pH}$ $\sim 5.5$ ) (Fig. S3), favoring the constant and specific oxygen supply to tumor tissue. $\mathrm{CaO}_{2}$ cores could not be dispersed in water, while the encapsulation of $\mathrm{SiO}_{2}$ shell allowed the $\mathrm{CaO}_{2}$ cores to be stably dispersed in aqueous media. Successful $\mathrm{SiO}_{2}$ shell encapsulation needs to control the water concentration in the ethanol solution containing $\mathrm{CaO}_{2}$ cores and tetraethyl orthosilicate (TEOS, $\mathrm{SiO}_{2}$ precursor) because high water concentration would lead to aggregation of $\mathrm{CaO}_{2}$ cores. Hence, concentrated $\mathrm{NaOH}$ solution instead of ammonia was used to catalyze the TEOS hydrolysis. Incorporation of DOX into shells was achieved by introducing DOX into the $\mathrm{SiO}_{2}$ shell growth media. With the constant DOX 
(a)
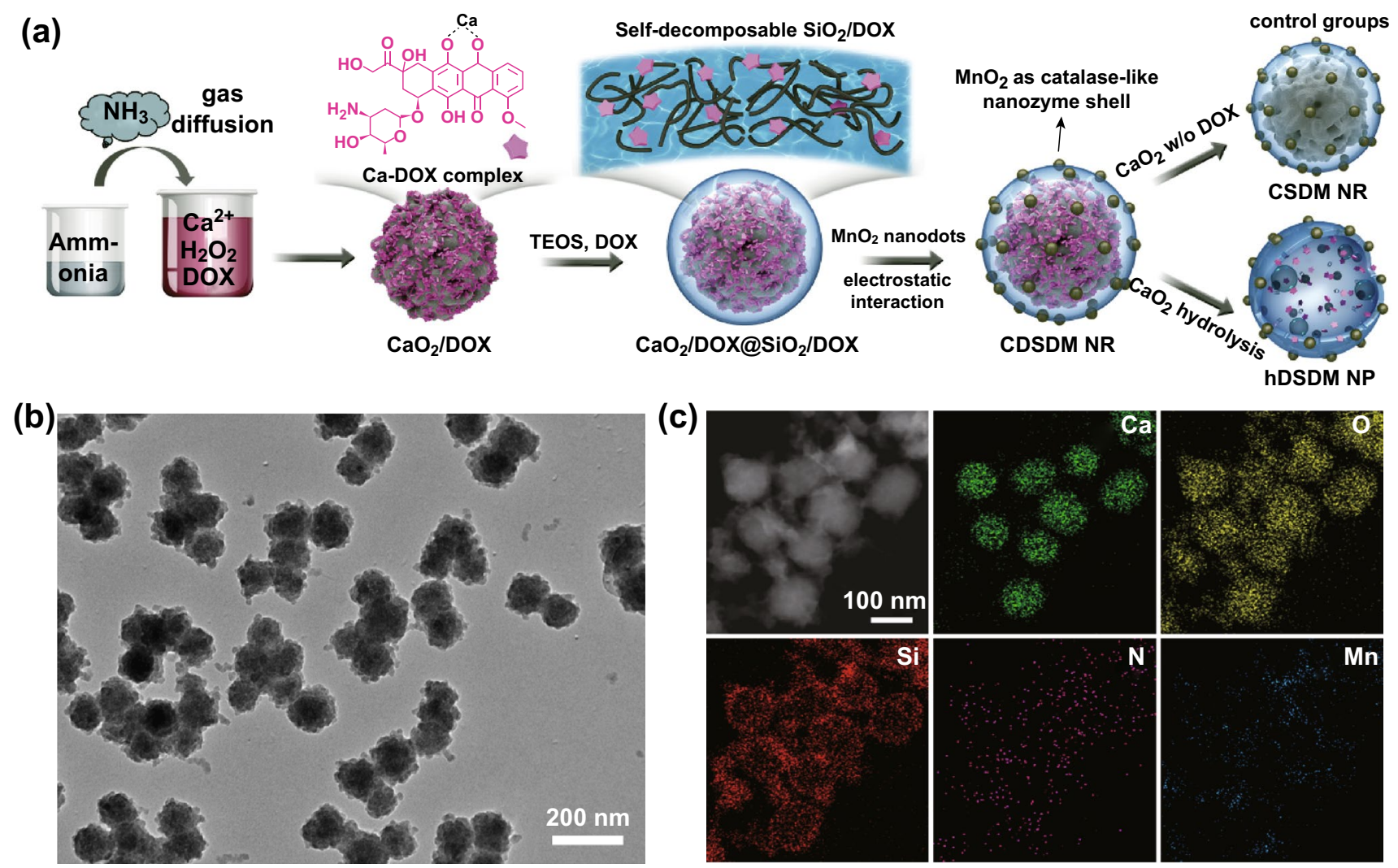

(c)
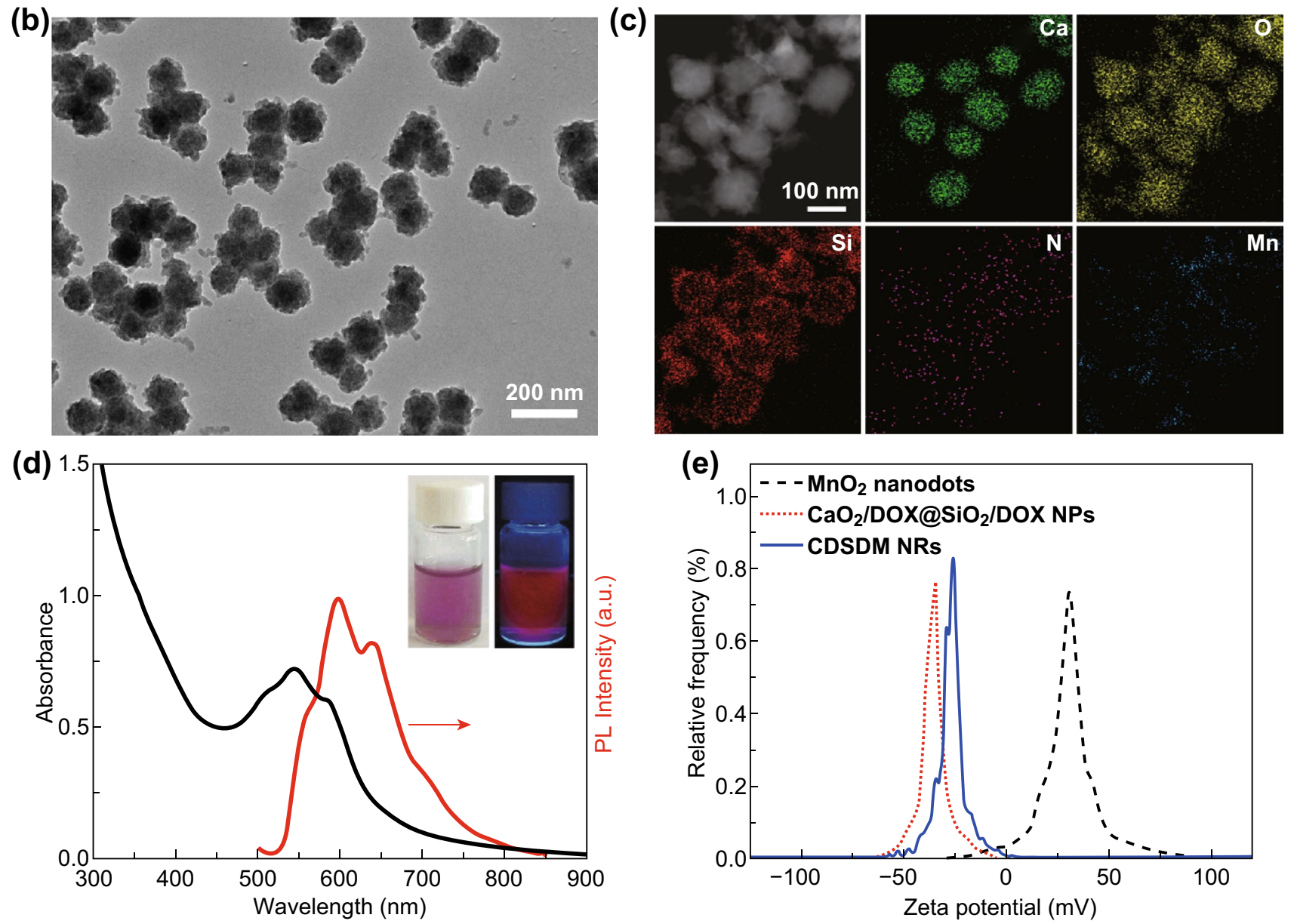

Fig. 1 a Schematic illustration of the preparation of CDSDM NRs. For the control groups, the $\mathrm{CaO}_{2} @ \mathrm{SiO}_{2} / \mathrm{DOX}-\mathrm{MnO}_{2}$ is named as CSDM NRs; after completely hydrolyzing $\mathrm{CaO}_{2}$ core in the CDSDM NRs, the hollow nanoparticle is named as hDSDM NPs. b Representative TEM image and $\mathbf{c}$ elemental mapping of CDSDM NRs. $\mathbf{d}$ UV-Vis absorption and photoluminescence spectra of CDSDM NRs. Insets are photographs of CDSDM NRs taken under (left) room light or (right) $365 \mathrm{~nm}$ light irradiation. e Zeta Potential of $\mathrm{MnO}_{2}$ nanodots, $\mathrm{CaO}_{2} / \mathrm{DOX}_{\mathrm{S}} \mathrm{SiO} / \mathrm{DOX}$ NPs and CDSDM NRs

release, the shell would collapse and decompose into pieces that can be excreted via renal system [40, 41]. After the optimization of shell thickness (Fig. S4), a $15 \mathrm{~nm}$ shell was selected for the subsequent study.
Ultra-small $(\sim 1.2 \mathrm{~nm}) \mathrm{MnO}_{2}$ nanodots were prepared according to the previous reports with certain modification (Fig. S5) [30], and were adsorbed onto the shells via charge attraction, after which the NR zeta potential increased from $-35 \mathrm{mV}$ to about $-26 \mathrm{mV}$. Considering the high catalytic 
efficacy of $\mathrm{MnO}_{2}$ toward $\mathrm{H}_{2} \mathrm{O}_{2}$, the adsorbed amount of $\mathrm{MnO}_{2}$ nanodots was limited to this extent to ensure the resulting NRs could be readily dispersed in water without flocculation in at least $24 \mathrm{~h}$ (Fig. S6). The resulting CDSDM NRs were about $125 \mathrm{~nm}$ in diameter (Fig. 1b). Energy-dispersive X-ray spectroscopy (EDS) mapping (Fig. 1c) shows the expected elements including $\mathrm{Ca}$ (from $\mathrm{CaO}_{2}$ cores), $\mathrm{Si}$ (from $\mathrm{SiO}_{2}$ shells), $\mathrm{O}$ (from cores and shells), $\mathrm{N}$ (from DOX) and $\mathrm{Mn}$ (from $\mathrm{MnO}_{2}$ nanodots). UV-Vis absorption and photoluminescence spectra (Figs. 1d, S7) show the DOX characteristic absorption and luminescence properties of CDSDM NRs. X-ray photoelectron spectroscopy (XPS) pattern (Fig. S8) also detected these elements, in which the relative weak Ca signal was ascribed to the limited detection depth of XPS (less than $10 \mathrm{~nm}$ ) than the typical shell thickness $(15 \mathrm{~nm})$, and further demonstrated the core/shell structure of the CDSDM NRs. The $\mathrm{Ca} / \mathrm{Si} / \mathrm{Mn}$ ratio in NRs measured using inductively coupled plasma optical emission spectrometry (ICP-OES) was about 1:0.91:0.1. Zeta potential of NRs in water is shown in Fig. 1e.

Incubating CDSDM NRs in phosphate buffer saline (PBS) led to the hydrolysis of $\mathrm{CaO}_{2}$ into $\mathrm{H}_{2} \mathrm{O}_{2}$, which diffused through the permeable $\mathrm{SiO}_{2}$ shell and decomposed in the presence of $\mathrm{MnO}_{2}$ nanodots to provide oxygen (Fig. 2a). The hydrolysis of $\mathrm{CaO}_{2}$ cores was faster at lower $\mathrm{pH}$ (Fig. $\mathrm{S} 3$ ), thus facilitated the controlled oxygen supply in acidic TME and endosome, as shown in Fig. 2b. Incubating NRs in $\mathrm{pH} 5.5 \mathrm{PBS}$ for about $4 \mathrm{~h}$ led to complete decomposition of cores, while the remained products were hollow nanoparticles (Fig. 2c, denoted as hDSDM NPs) with about $48 \%$ of the weight of whole NRs. The similar zeta potential suggested that $\mathrm{MnO}_{2}$ nanodots were basically retained in these hDSDM NPs. Due to the incorporation of DOX molecules, the cross-linking degree of silica matrix (products of TEOS hydrolysis) would be lowered due to the steric effect of DOX molecules. In aqueous media where DOX is soluble, the constant release of DOX from the silica will trigger the silica shell disruption and finally lead to the complete fragmentation into poly(silicic acid) that can be excreted via renal system (Fig. 2c) [41]. $\mathrm{MnO}_{2}$ nanodots were also decomposed after about $24 \mathrm{~h}$ of incubation, in accordance with previous report [29]. The self-decomposition capability of NRs was believed to be important for reducing systemic toxicity when used in vivo.

Incubating hypoxic B16F10 cells with CDSDM NRs for $4 \mathrm{~h}$, the cellular hypoxia was effectively reconditioned
(Fig. 2d) due to the constant oxygen supply by CDSDM NRs. In obvious comparison, the hDSDM NPs exhibited no such effect, suggesting the importance of $\mathrm{CaO}_{2}$ cores in providing sufficient oxygen; $\mathrm{CDSD}\left(\mathrm{CaO}_{2} / \mathrm{DOX} @ \mathrm{SiO}_{2} /\right.$ DOX) NP treatment also showed no obvious hypoxia relief, which should be due to the limited $\mathrm{H}_{2} \mathrm{O}_{2}$ decomposition for oxygenation without $\mathrm{MnO}_{2}$ nanodots. The cytoplasmic distribution of the main DOX fluorescence suggested that DOX might be trapped inside shells at the beginning despite the decomposition of $\mathrm{CaO}_{2}$, because otherwise substantial DOX would be accumulated in nuclei. This was confirmed by analyzing the release profile via UV-Vis absorption measurement that the DOX release was lagged much behind the oxygen generation (Fig. 2b, e). Meanwhile, lower $\mathrm{pH}$ allowed faster DOX release. The DOX release from $\mathrm{SiO}_{2}$ shells was found to be more sustained by analyzing CSDM NRs (absorption spectra shown in Fig. S9) that contained no DOX in $\mathrm{CaO}_{2}$ cores, in line with the lower decomposition rate of $\mathrm{SiO}_{2}$ shells than $\mathrm{CaO}_{2}$ cores. In vitro antitumor capabilities of CDSDM NRs, CSDM NRs and hDSDM NPs were evaluated via MTT assay (Fig. S10). The results showed that CDSDM NRs exhibited significantly higher cytotoxicity than hDSDM NPs under hypoxic condition. This was ascribed to the sensitization of chemotherapy by oxygenation, which has become a widely explored strategy for improving therapeutic efficacy of chemo drugs.

\subsection{Relief of Tumor Hypoxia and Suppression of Hypoxia-Adenosinergic Signaling}

Lower oxygen tension in solid tumor tissue can weaken the efficacy of spontaneous or adoptive immunotherapy, in which the hypoxia-adenosinergic signaling has been known as a master pathway [12]. In this work, the capability of CDSDM NRs in reversing such immunosuppression was tested. In vivo distribution of CDSDM NRs when intravenously injected into mice-bearing B16F10 melanoma tumor under armpit was first studied. By monitoring the fluorescence signal of DOX, substantial intratumoral accumulation was observed 8-h post-injection (Fig. 3a) owing to the enhanced permeability and retention effect of tumor vasculature [42]. Similar results were observed from ex vivo imaging of excised organs and microscopic imaging of histological sections (Figs. 3a, S11). Quantitative analysis using inductively coupled plasma mass 

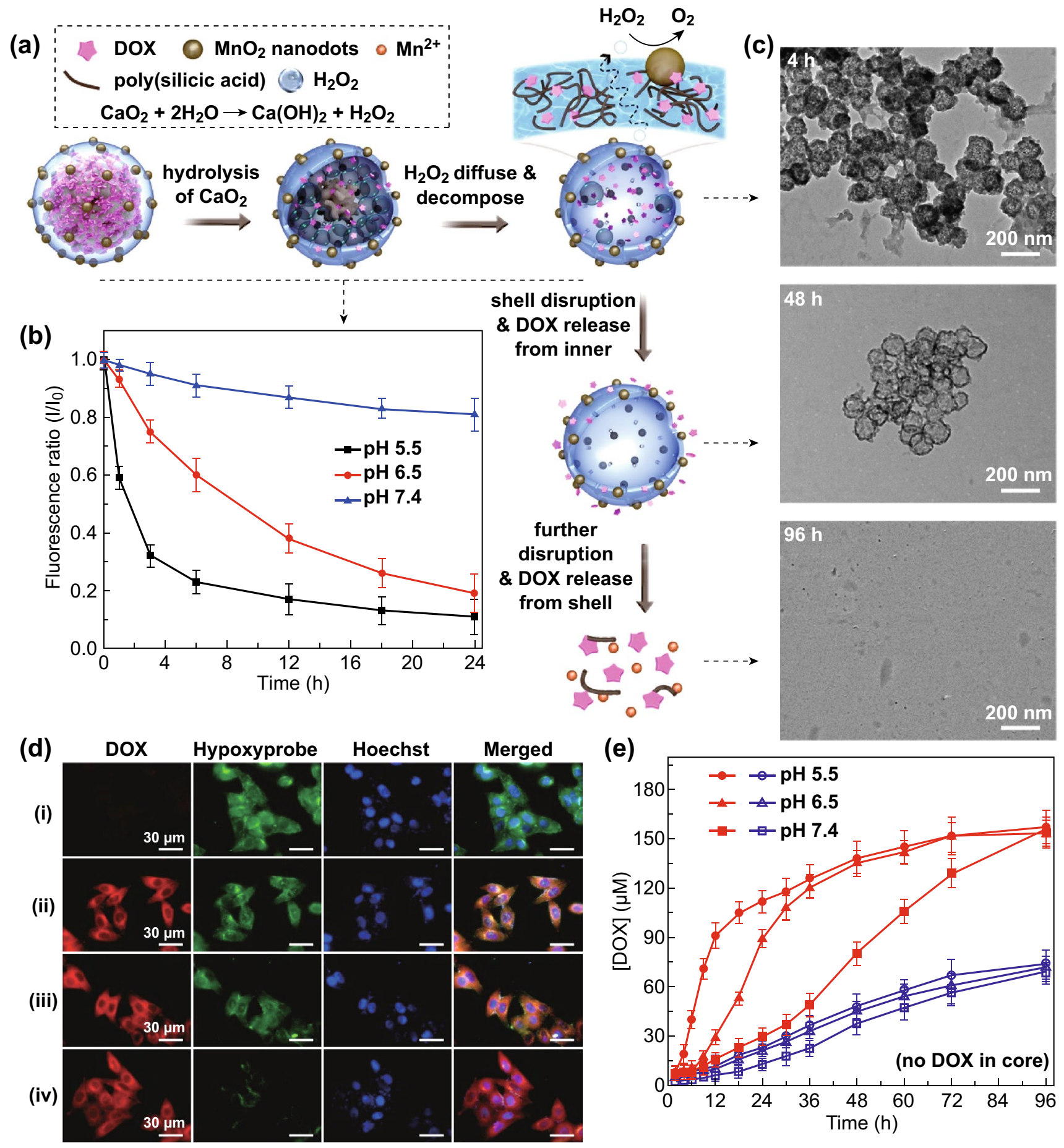

Fig. 2 a Schematic illustration of the processes of oxygen generation, DOX release and decomposition of NRs. b The measurement of oxygen release process of CDSDM NRs, shown as the ratio of fluorescence intensity of $\mathrm{Ru}(\mathrm{dpp})_{3} \mathrm{Cl}_{2}$ (an oxygen indicator) after incubated with $\mathrm{NRs}$ for different durations $(I)$ to that before incubation $\left(I_{0}\right)$. $\mathbf{c}$ TEM images of the decomposition process of CDSDM NRs after incubated in pH 5.5 PBS for different durations. d Representative CLSM images of B16F10 cells from hypoxia cultivation after co-incubated with (1) PBS, (2) hDSDM NPs, (3) CDSD (without $\mathrm{MnO}_{2}$ nanodots) NPs and (4) CDSDM NRs for $4 \mathrm{~h}$. Hypoxia level was detected using Hypoxyprobe. e DOX release from CDSDM NRs and CSDM NRs in PBS of different $\mathrm{pH}$ values 
spectrometry (ICP-MS) showed up to $23 \%$ of $\mathrm{Si}$ accumulation in tumor 8-h post-injection (Fig. S12). Next, intratumoral hypoxia level was evaluated using hypoxia marker Hypoxyprobe. As shown in Fig. 3b, the intratumoral hypoxia was clearly alleviated even after one dose of CDSDM NR administration, in contrast to hDSDM NP treatment. Three-dimensional cell culture model (Fig. S13) and photoacoustic imaging (Fig. S14) were also employed to validate the hypoxia alleviation, and the results provided further demonstration of the superior hypoxia-relieving capability of CDSDM NRs than hDSDM NPs. Although the $\mathrm{MnO}_{2}$ nanodots on hDSDM NPs can catalyze the decomposition of $\mathrm{H}_{2} \mathrm{O}_{2}$, which is relatively abundant in tumor tissue [29, 43], they seem to be much less efficient in alleviating tumor hypoxia, further underscoring the necessity of $\mathrm{CaO}_{2}$ cores in this work.

Next, the effect of CDSDM NR treatment on hypoxiaadenosinergic signaling was studied. Expressions of several key molecular events of the signaling including hypoxiainducible factor- $1 \alpha$ (HIF-1 $\alpha$ ), CD39/CD73 and adenosine were tested. Flow cytometry and western blot results showed that treatment with CDSDM NRs resulted in significantly reduced expression of HIF- $1 \alpha$ in cells from tumor tissue (Fig. 3c) and CD39/CD73 in T cells (Fig. 3d), respectively, compared with the hDSDM group. HIF- $1 \alpha$ is the master regulator of hypoxic response in T cells, while the CD39/ CD73 expression can be lowered by HIF- $1 \alpha$ reduction [44, 45]; these should be ascribable to the reduced CD39/CD73 expression in T cells by hypoxia relief. HIF- $1 \alpha$ can be rapidly degraded under normal conditions via being poly-ubiquitinated, while hypoxia (oxygen tension lower than about $3 \%$ ) can drive the enzyme-mediating HIF- $1 \alpha$ degradation nonfunctional [46]. Therefore, the reduced HIF-1 $\alpha$ was also a further demonstration of the relief of hypoxia. The extracellular adenosine in TME also significantly decreased largely as a consequence of the reduced expression of adenosine generating CD39/CD73 (Fig. 3e). Taken together, it can be concluded that the hypoxia-adenosinergic signaling has been inhibited efficiently, through which a positive immune modulation can be expected via its effects on Tregs and cytotoxic T cells (CTL) [12].

\subsection{Strong Tumor Regression with Oxygen-Self-Generated CDSDM NRs}

Given the effective inhibition of hypoxia-adenosinergic signaling, one can expect a synergistic effect between NR treatment and immunotherapy. CTLA-4 blockade, a FDA approved immune checkpoint-blocking antibody for the treatment of advanced melanoma [47], was used for the eradication of B16F10 melanoma tumor in this work. To reduce the damage of DOX-containing NRs to the immune system, CSDM NRs was also used for adaptive administration with CDSDM NRs (Fig. 4a). The DOX amount in CSDM NRs was about half of that in CDSDM NRs (Table S1). Adaptive administration here means adjusting the dosage of chemotherapeutic drug according to the variation of tumor size rather than giving an invariable high dose in order to protect the immune system by chemo agent (DOX) [48, 49].

In this work, CDSDM NRs were given in initial treatment, while CSDM NRs would be used as alternatives if tumor volume decreased or increased less than $10 \%$ from the previous measurement; otherwise CDSDM NRs would be reused. In a 20-day treatment period, different agents were intravenously injected every 2 days, while CTLA-4 blocking antibody was injected intraperitoneally every 4 days. Tumor volume, body weight and survival rate in each group were monitored during and after treatment (Fig. 4b-d). Compared with the oxygen-self-produced CDSDM NRs + CTLA-4 blockade, the results showed that hDSDM NPs + CTLA-4 blockade showed much weaker antitumor efficacy (evaluated according to tumor volume, survival rate and histopathology staining of tumor tissue, Figs. 4b, d and S15, S16), although stronger than CTLA-4 blockade alone. Therefore, relief of intratumoral hypoxia should be a prerequisite for CTLA-4 blockade to exert due antitumor efficacy, considering the same DOX concentration in hDSDM NPs and CDSDM NRs. Meanwhile, adaptive administration of CDSDM/ CSDM NRs showed stronger efficacy than the CDSDM NRs albeit the lower total DOX dose (Fig. S17), suggesting an immune benefit of this chemotherapy strategy [49]. In a control study using immune-deficient nude mice, the synergistic effects between CDSDM NR and CTLA-4 blockade treatment were much limited due to the attenuated cellular immunity (Fig. S19). Instead, the DOX in CDSDM NRs accounted for the observed antitumor efficacy. Therefore, it is believed that a complete immune system is a prerequisite 

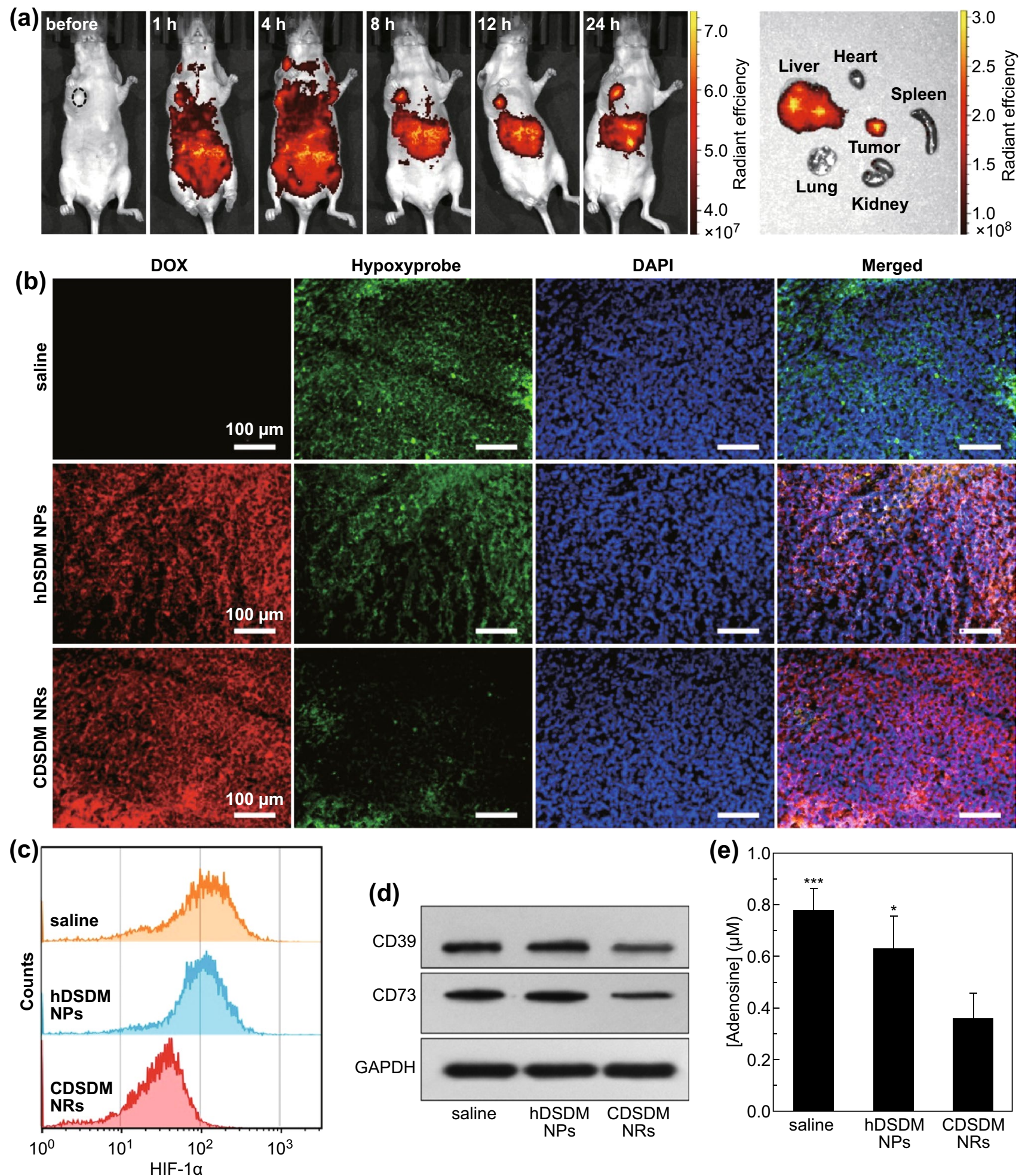

Fig. 3 a In vivo fluorescence monitoring of the distribution of CDSDM NRs in B16F10 tumor-bearing mice at scheduled time points postintravenous injection, and ex vivo fluorescence of major organs and tumor 8-h post-injection. b Fluorescence imaging of tumor tissue sections after receiving different treatments, in which signals include DOX fluorescence, Hypoxyprobe (hypoxia indicator) and DAPI. c Flow cytometric analysis of the intratumoral HIF-1 $\alpha$ level after different treatments. d Western blot results of the expressions of CD39 and CD73 on intratumoral $\mathrm{T}$ lymphocytes after 2 days of the indicated treatments. e Concentrations of extracellular adenosine in tumor tissue after 2 days of the indicated treatments. $* P<0.05 ; * * * P<0.001$ versus the last group 
(a)
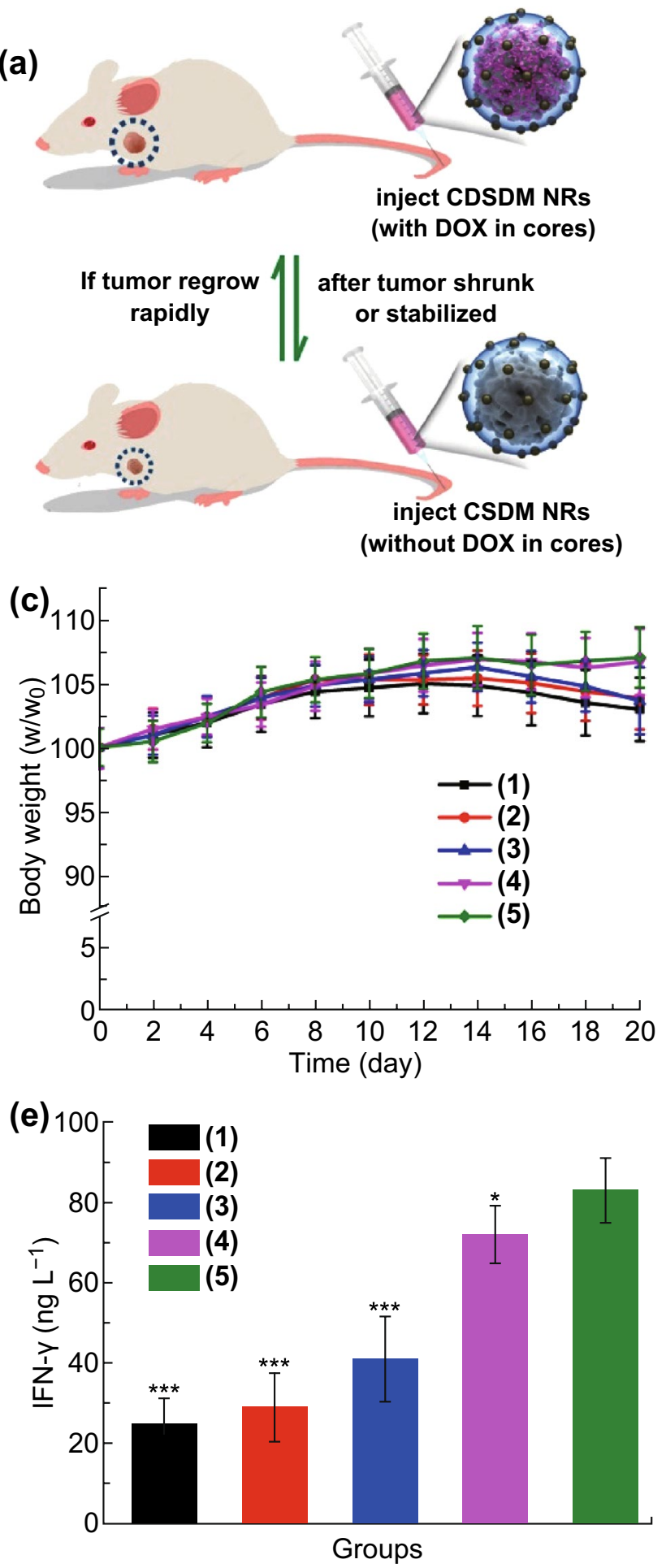

(b)

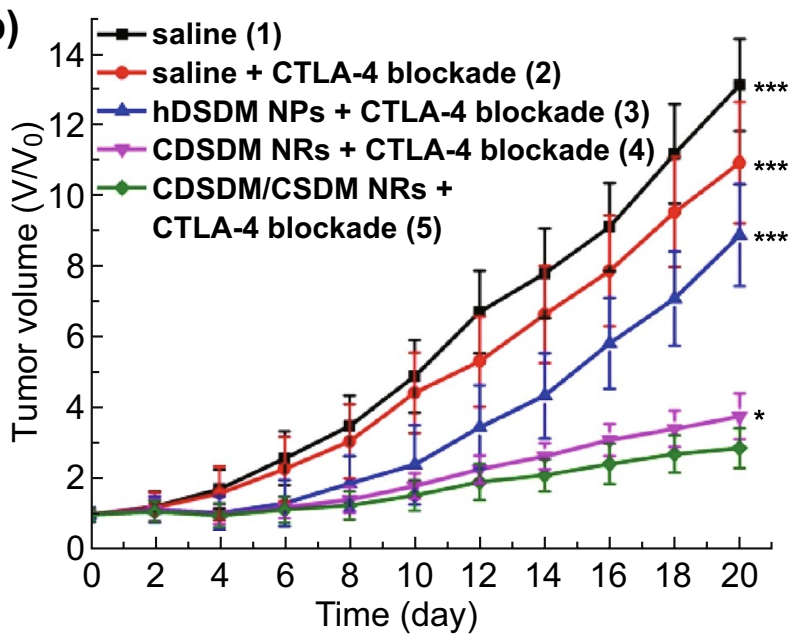

(d)
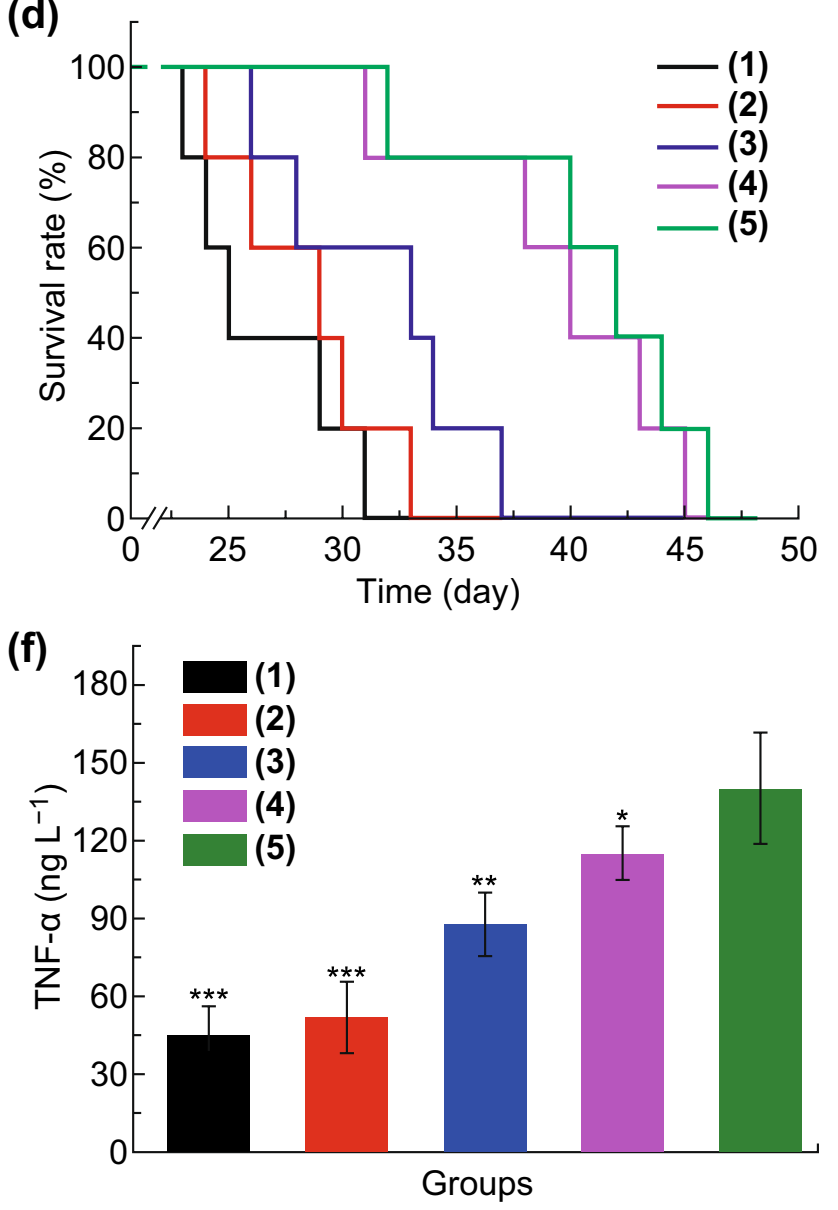

Fig. 4 a Illustration of adaptive administration of CDSDM NRs and CSDM NRs. b Relative tumor volumes and $\mathbf{c}$ body weights of B16F10 tumor-bearing mice received with different treatments during the treatment period. d Survival rate of mice in each group during and after treatment. Serum levels of $\mathbf{e}$ IFN- $\gamma$ and $\mathbf{f}$ TNF- $\alpha$ detected after 8 days of different treatments. $* P<0.05 ; * * P<0.01$; *** $P<0.001$ versus Group 5 
for the sensitization of immunotherapy by CDSDM NRinduced hypoxia relief.

We also tested the immune effect in molecular level by measuring the production of typical cytokines. Measurement of the serum levels of IFN- $\gamma$ and TNF- $\alpha$ (typical markers of cellular immune response) showed increased production in mice treated with CDSDM NRs than that with hDSDM NPs (Fig. 4e, f), which should be one of the results of the alleviation of hypoxia-adenosinergic signaling and reflected a stronger antitumor immune response [50, 51]. Adaptive administration of CDSDM and CSDM NRs further induced slight increase in cytokine production due to the more potent immune system with reduced DOX dose.

Meanwhile, no obvious reduction in body weight was observed in mice treated with CDSDM/CSDM NRs (Fig. 4c), suggesting the indistinctive systemic toxicity. The body weight loss in mice in other three groups could be ascribed to reduced food intake and fat loss due to the increased tumor burden. Hematoxylin-eosin histopathology staining results also indicated the rather limited damage of major organs caused by CDSDM/CSDM NRs (Fig. S20). Hemolysis test was also performed by incubating CDSDM NRs with red blood cells, and the hemolysis induced by CDSDM NRs at the concentration for treatment was believed to be acceptable (Fig. S21). [52] Therefore, the asprepared oxygen-self-produced CDSDM (and CSDM) NRs were considered to be a considerably safe hypoxia-relieving nanoformulation.

\subsection{Enhanced $\mathrm{CD8}^{+} \mathrm{T}$ Cell Infiltration and Suppressed Treg Development}

Tregs can diminish spontaneous or blockade-mediated antitumor immune responses via the expressions of CD39/CD73 and constitutive CTLA-4 [53]. The former could deliver negative immunomodulation via hypoxia-adenosinergic signaling, while the latter has made Treg a master target of the currently widely studied CTLA-4 blockade [47, 53]. It has been proposed that hypoxia alleviation by hyperoxia breathing could reduce the Treg population [19], and in this work, the effect of oxygen-self-produced NRs on Treg population was studied. Flow cytometric results showed that in comparison with hDSDM NPs, treatment with CDSDM or CSDM NRs resulted in a significant decrease (by a factor of $\sim 4$ ) in intratumoral Treg $\left(\mathrm{CD} 4^{+} \mathrm{CD} 25^{+}\right.$Foxp $\left.^{+}\right)$population (Fig. 5a). In line with the superior tumor regression using CDSDM NRs than hDSDM NPs, this suggested that Treg-related immunosuppression could be greatly alleviated in complementary manners with the inhibition of hypoxia-adenosinergic signaling and the use of CTLA-4 blockade (CTLA- 4 contributes to the immunosuppressive function of Tregs). Also, it can be expected to achieve synergistic effect when combining NRs with other blocking antibodies of immune checkpoints, for instance the PD-1/PD-L1 [54].

Antitumor T cells can be repelled in hypoxic areas, and this is why endogenous developed or adoptively transferred tumor-responsive $\mathrm{CD}^{+} \mathrm{T}$ cells showed limited antitumor efficacy and were often combined with other agents such as antagonist of adenosine receptor [55]. Inspired by the observation that the recruitment of $\mathrm{CD}^{+} \mathrm{T}$ cells could be enhanced by respiratory hyperoxia [19], we tested whether the hypoxia relief with CDSDM NRs facilitated the intratumoral infiltration of antitumor $\mathrm{CD} 8^{+} \mathrm{T}$ cells. Tumor tissues from different groups were analyzed by the co-staining of HIF- $1 \alpha$ and CD8, and the results suggested that in both shallow (Fig. S22) and deep (Fig. 5b) tumor tissues, treatment with CDSDM NRs resulted in reduced HIF-1 $\alpha$ expression and enhanced intratumoral accumulation and infiltration of $\mathrm{CD}^{+} \mathrm{T}$ cells. Therefore, hypoxia alleviation with our NRs could significantly reduce the repelling of CTLs (i.e., activated $\mathrm{CD} 8^{+} \mathrm{T}$ cells) by tumor tissue, and as a desirable extension of the functions of CDSDM NR treatment, this was ascribed to another contribution to the enhanced tumor regression as shown in Fig. 4b.

The released DOX from CDSDM NRs, while serving as the typical chemo agent to induce apoptosis of tumor cells, was also found to be immunogenic. Incorporation of DOX into $\mathrm{SiO}_{2}$ shells were designed to achieve immuno-chemocombined therapy and to enable the self-decomposition of the $\mathrm{SiO}_{2}$ shells; meanwhile, it has been reported that anthracyclines, in particular, DOX-treated dying or apoptotic tumor cells can serve as in situ vaccine to induce immunogenic tumor cell death in the absence of any adjuvant or co-stimulus [56, 57]. Therefore, we have explored the effect of DOX on immunogenicity by analyzing maturation of dendritic cells (DCs), which can phagocytose the DOX-treated apoptotic cells, present the neoantigens to T cells and mature during this process. DCs were isolated from axillary lymph nodes at tumor side after 4 days of different treatments, and flow cytometric results of the staining of CD80 and CD86 (typical markers of DC maturation) showed that the 

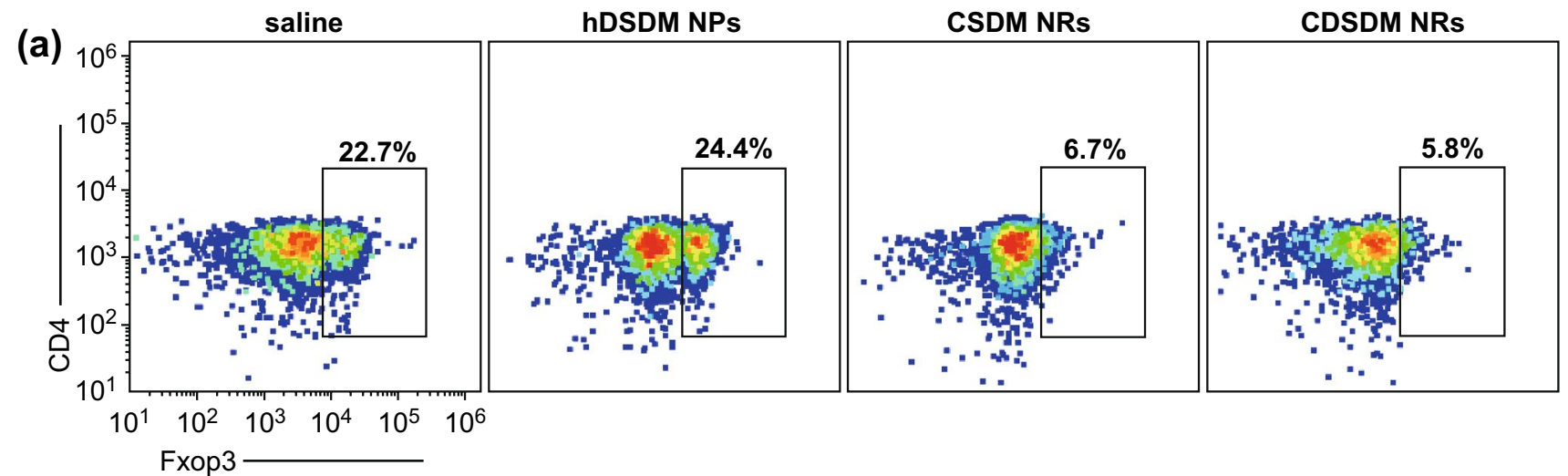

(b)
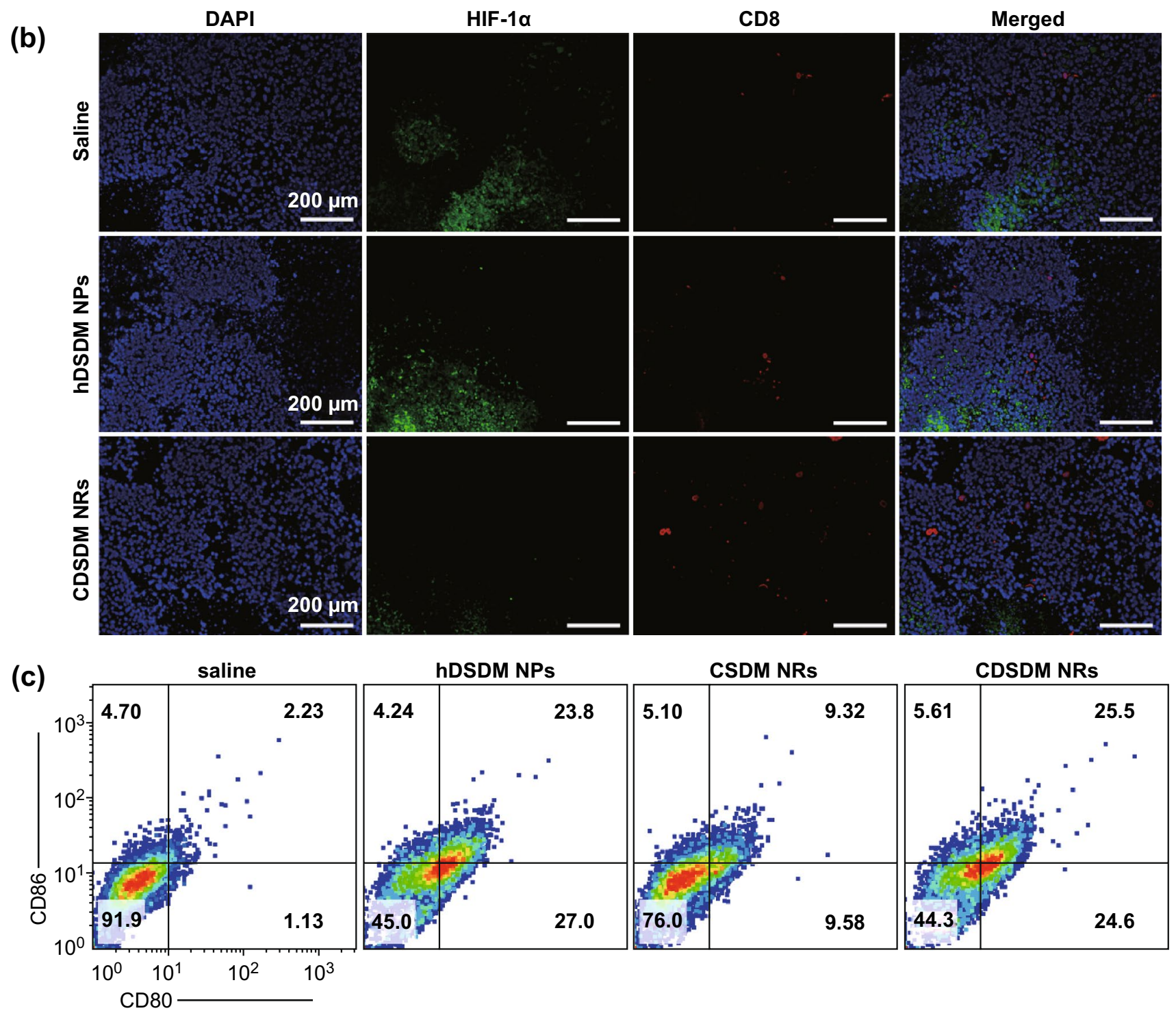

Fig. 5 a Flow cytometry plots showing the percentages of intratumoral Treg (gated on CD25 $\mathrm{T}$ cells) populations from mice after 4 days of the indicated treatments. b Immunofluorescence results of the co-staining of HIF-1 $\alpha$ and CD8 to deep ( $>2$ mm from the surface) tumor tissues from mice received 4 days of the indicated treatments. $\mathbf{c}$ Flow cytometry plots showing the percentages of CD80 ${ }^{+}$and CD86 ${ }^{+}$matured DCs $($gated on CD11c+ DCs) from the axillary lymph nodes of mice after 4 days of the indicated treatments 
percentage of matured DCs was much higher in mice treated with hDSDM NPs or CDSDM NRs (Fig. 5c). The increase in median percentage in CSDM NR group was ascribed to the lower DOX concentration. Therefore, in current experimental setting, the DOX embedded in $\mathrm{SiO}_{2}$ shells served not only as the chemotherapeutic drug but also a vaccine primer that led to a productive immune activation, together with CTLA-4 blockade in a synergistic manner (CTLA-4 blockade can enhance the immunopermissive recognition between naive $\mathrm{T}$ cells and antigen-presenting mature DCs) [58].
Based on the above observations, the schematic illustration of the proposed roles of CDSDM NRs in tumor hypoxia relief is shown in Scheme 1a. Both the hydrolysis of $\mathrm{CaO}_{2}$ and the endogenous $\mathrm{H}_{2} \mathrm{O}_{2}$ within tumor contributed the abundant oxygenation via the catalase-like activity of $\mathrm{MnO}_{2}$ nanodots. The multiple roles of CDSDM NRs in tumor rejection are shown in Scheme 1b. Basically, the immunopriming effects of CDSDM NRs were derived from their two capabilities, i.e., the TME-motivated oxygen-self-production, and the DOX releasing during oxygen production and

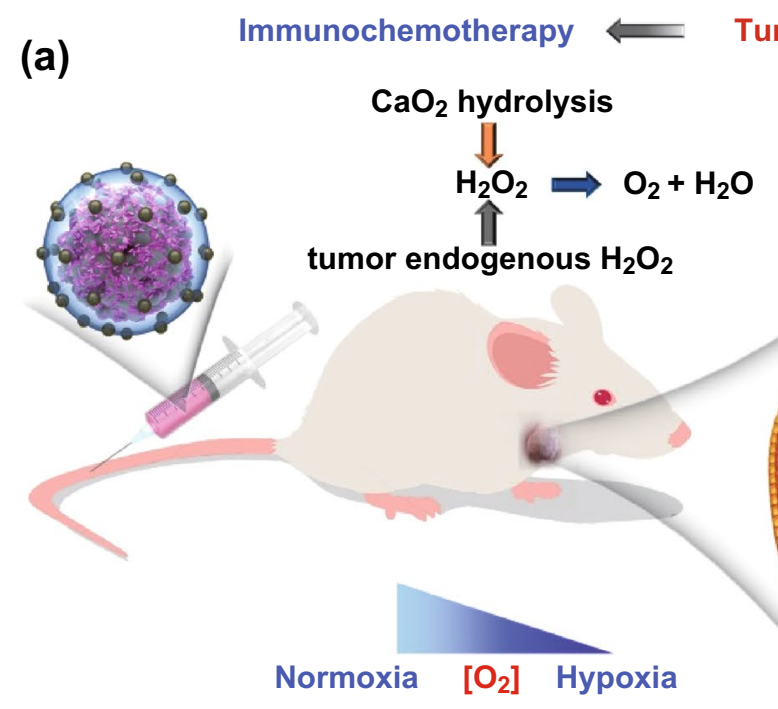

Tumor Hypoxia Relief $\quad \Longrightarrow$ Immunosupportive TME

(a)

(b)

Sensitized Cancer Immunochemotherapy

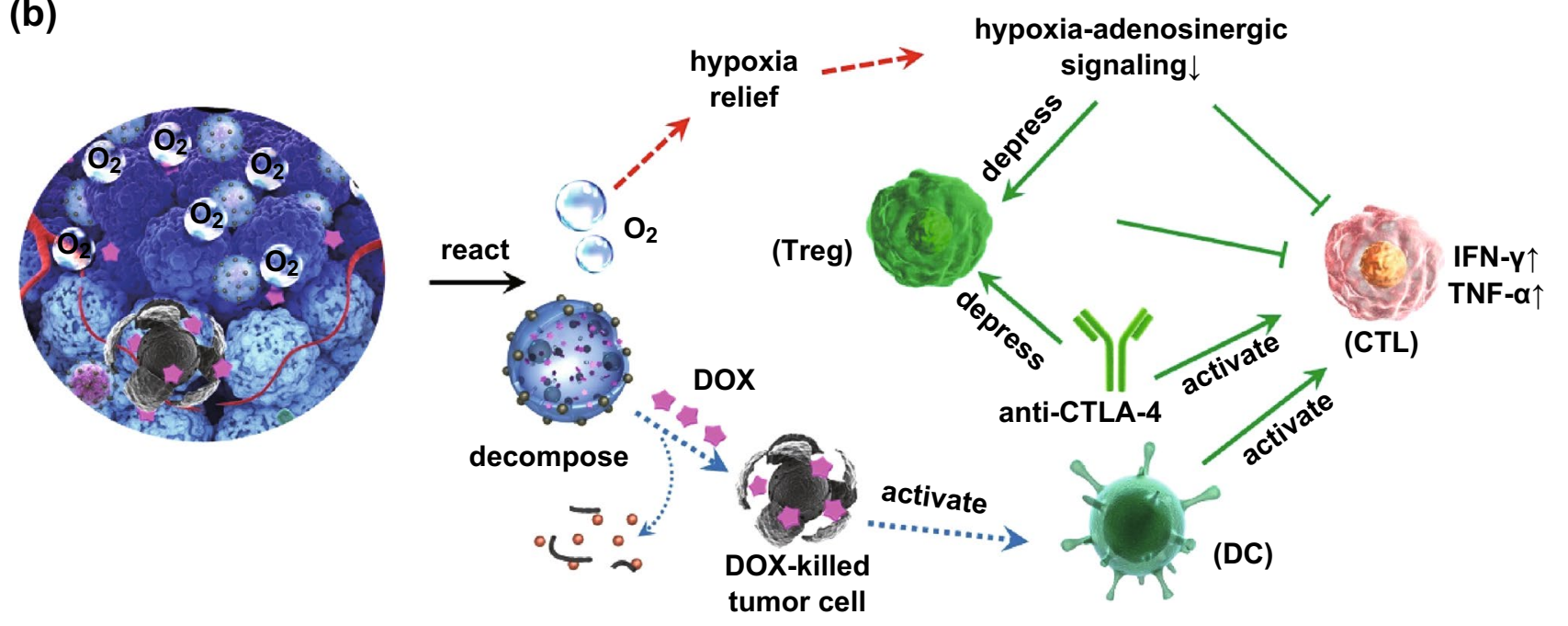

Scheme 1 a Schematic illustration of the proposed roles of CDSDM NRs in tumor hypoxia relief. b The proposed mechanism and reaction pathways include the relief of hypoxia-induced immunosuppression (red dashed arrows), the DOX-mediated chemotherapy and immune activation (blue dotted arrows), and the synergistic actions between them and the CTLA-4 blockade (green arrows). (Color figure online) 
NR decomposition. The former effectively relieved intratumoral hypoxia, and the resulting immune benefits included: (1) the immunosuppressive hypoxia-adenosinergic signaling was inhibited (Fig. 3b-e), and as a direct result, the production of antitumor cytokines (e.g., IFN- $\gamma$ and TNF- $\alpha$ ) by $\mathrm{T}$ lymphocytes increased (Fig. 4e, f). (2) The negative immunomodulation by Tregs was inhibited due to the decreased expression of CD39/CD73 (Fig. 3d) and the reduced population (Fig. 5a). These effects could promote the activation of antitumor immune responses in a complementary manner with the CTLA-4 blockade. (Treg is a master expression host for CTLA-4). (3) Due to the decreased hypoxic repelling effect, the intratumoral infiltration and accumulation of $\mathrm{CD}^{+} \mathrm{T}$ cells were improved (Fig. 5b). The released DOX from CDSDM NRs, while serving as the typical chemo agent to induce apoptosis of tumor cells, was also found to be immunogenic by inducing DC maturation (Fig. 5c) and thereby promoting $\mathrm{T}$ cell activation synergistically with CTLA-4 blockade.

\section{Conclusion}

In summary, a self-oxygenation/degradable inorganic nanozyme system has been fabricated to relieve tumor hypoxia for cancer immunochemotherapy. By integrating $\mathrm{CaO}_{2}$ core as the oxygen-storing component, our system provides improved oxygenation and capability of relieving hypoxia compared with the oxygenation methods that only decompose the endogenous $\mathrm{H}_{2} \mathrm{O}_{2}$ in TME. Compared to the reported design that directly delivers $\mathrm{H}_{2} \mathrm{O}_{2}$, our strategy shows acid TME-preferred oxygenation and enhanced physiological stability. In vivo tests validated that the CDSDM NRs could successfully relieve hypoxia in mice-bearing B16F10 tumor, thus reversing the immunosuppressive TME to favor antitumor immune response. Consequently, the CTLA-4-mediated immunotherapy was greatly sensitized. Further studies revealed that CDSDM NR treatment also enhanced the intratumoral infiltration of $\mathrm{CD}^{+} \mathrm{T}$ cells and decreased the population of immunosuppressive Tregs. Meanwhile, the DOX in CDSDM NRs also elicited desirable maturation of DCs, further favored a productive antitumor immune response. Overall, a novel self-oxygenation/degradable nanozyme system has been proposed to improve the cancer immunochemotherapy via relieving tumor hypoxia without causing notable toxic side effects. This strategy not only shows promise in relieving tumor hypoxia and sensitizing cancer immunochemotherapy, but also holds the potential for applications in oxygen-favored cancer therapy (i.e., photodynamic and radiation therapy) and oxygen deficiencyoriginated diseases.

Acknowledgements This work was financially supported by the National Natural Science Foundation of China (Nos. 81371627 and 81727804), the Jiangsu Provincial Natural Science Fund for Distinguished Young Scholars (BK201900), and the "Double First-Class" University project (Nos. CPU2018GY24 and CPU2018GY20). Prof. Cheng acknowledges the support of the DRS POINT Fellowship, Alexander von Humboldt Fellowship, and Thousand Youth Talents Plan.

Open Access This article is distributed under the terms of the Creative Commons Attribution 4.0 International License (http:// creativecommons.org/licenses/by/4.0/), which permits unrestricted use, distribution, and reproduction in any medium, provided you give appropriate credit to the original author(s) and the source, provide a link to the Creative Commons license, and indicate if changes were made.

Electronic supplementary material The online version of this article (https://doi.org/10.1007/s40820-019-0305-x) contains supplementary material, which is available to authorized users.

\section{References}

1. H. Wang, D.J. Mooney, Biomaterial-assisted targeted modulation of immune cells in cancer treatment. Nat. Mater. 17(9), 761-772 (2018). https://doi.org/10.1038/s41563-018-0147-9

2. Y. Chen, R. Xia, Y. Huang, W. Zhao, J. Li et al., An immunostimulatory dual-functional nanocarrier that improves cancer immunochemotherapy. Nat. Commun. 7, 13443 (2016). https://doi.org/10.1038/ncomms 13443

3. P. Sharma, S. Hu-Lieskovan, J.A. Wargo, A. Ribas, Primary, adaptive, and acquired resistance to cancer immunotherapy. Cell 168(4), 707-723 (2017). https://doi.org/10.1016/j. cell.2017.01.017

4. D.F. Quail, J.A. Joyce, Microenvironmental regulation of tumor progression and metastasis. Nat. Med. 19, 1423 (2013). https://doi.org/10.1038/nm.3394

5. J.-N. Liu, W. Bu, J. Shi, Chemical design and synthesis of functionalized probes for imaging and treating tumor hypoxia. Chem. Rev. 117(9), 6160-6224 (2017). https://doi. org/10.1021/acs.chemrev.6b00525

6. N. Li, L. Yu, J. Wang, X. Gao, Y. Chen, W. Pan, B. Tang, A mitochondria-targeted nanoradiosensitizer activating reactive oxygen species burst for enhanced radiation therapy. Chem. Sci. 9(12), 3159-3164 (2018). https://doi.org/10.1039/C7SC0 $4458 \mathrm{E}$ 
7. D. Guo, X. Ji, F. Peng, Y. Zhong, B. Chu, Y. Su, Y. He, Photostable and biocompatible fluorescent silicon nanoparticles for imaging-guided co-delivery of sirna and doxorubicin to drug-resistant cancer cells. Nano-Micro Lett. 11, 27 (2019). https://doi.org/10.1007/s40820-019-0257-1

8. D. Huo, S. Liu, C. Zhang, J. He, Z. Zhou, H. Zhang, Y. Hu, Hypoxia-targeting, tumor microenvironment responsive nanocluster bomb for radical-enhanced radiotherapy. ACS Nano 11(10), 10159-10174 (2017). https://doi.org/10.1021/acsna no. $7 \mathrm{~b} 04737$

9. D. Lukashev, B. Klebanov, H. Kojima, A. Grinberg, A. Ohta et al., Cutting edge: hypoxia-inducible factor $1 \alpha$ and its activation-inducible short isoform I. 1 negatively regulate functions of $\mathrm{CD}^{+}$and $\mathrm{CD}^{+}{ }^{+}$lymphocytes. J. Immunol. 177(8), 49624965 (2006). https://doi.org/10.4049/jimmunol.177.8.4962

10. A. Ohta, R. Diwanji, R. Kini, M. Subramanian, A. Ohta, M. Sitkovsky, In vivo $\mathrm{T}$ cell activation in lymphoid tissues is inhibited in the oxygen-poor microenvironment. Front. Immunol. 2, 27 (2011). https://doi.org/10.3389/fimmu.2011.00027

11. R.K. Jain, Antiangiogenesis strategies revisited: from starving tumors to alleviating hypoxia. Cancer Cell 26(5), 605-622 (2014). https://doi.org/10.1016/j.ccell.2014.10.006

12. M.V. Sitkovsky, S. Hatfield, R. Abbott, B. Belikoff, D. Lukashev, A. Ohta, Hostile, hypoxia-A2-adenosinergic tumor biology as the next barrier to overcome for tumor immunologists. Cancer Immunol. Res. 2(7), 598-605 (2014). https://doi. org/10.1158/2326-6066.Cir-14-0075

13. W.R. Wilson, M.P. Hay, Targeting hypoxia in cancer therapy. Nat. Rev. Cancer 11, 393 (2011). https://doi.org/10.1038/nrc30 64

14. J. Liu, L. Tian, R. Zhang, Z. Dong, H. Wang, Z. Liu, Collagenase-encapsulated ph-responsive nanoscale coordination polymers for tumor microenvironment modulation and enhanced photodynamic nanomedicine. ACS Appl. Mater. Interfaces 10(50), 43493-43502 (2018). https://doi. org/10.1021/acsami.8b17684

15. Y. Huang, S. Goel, D.G. Duda, D. Fukumura, R.K. Jain, Vascular normalization as an emerging strategy to enhance cancer immunotherapy. Cancer Res. 73(10), 2943-2948 (2013). https ://doi.org/10.1158/0008-5472.Can-12-4354

16. Z. Zhou, B. Zhang, S. Wang, W. Zai, A. Yuan, Y. Hu, J. Wu, Perfluorocarbon nanoparticles mediated platelet blocking disrupt vascular barriers to improve the efficacy of oxygen-sensitive antitumor drugs. Small 14(45), 1801694 (2018). https ://doi.org/10.1002/smll.201801694

17. M. Thiel, A. Chouker, A. Ohta, E. Jackson, C. Caldwell et al., Oxygenation inhibits the physiological tissue-protecting mechanism and thereby exacerbates acute inflammatory lung injury. PLoS Biol. 3(6), e174 (2005). https://doi.org/10.1371/journ al.pbio.0030174

18. S.M. Hatfield, J. Kjaergaard, D. Lukashev, B. Belikoff, T.H. Schreiber et al., Systemic oxygenation weakens the hypoxia and hypoxia inducible factor $1 \alpha$-dependent and extracellular adenosine-mediated tumor protection. J. Mol. Med. 92(12), 1283-1292 (2014). https://doi.org/10.1007/s0010 9-014-1189-3
19. S.M. Hatfield, J. Kjaergaard, D. Lukashev, T.H. Schreiber, B. Belikoff et al., Immunological mechanisms of the antitumor effects of supplemental oxygenation. Sci. Transl. Med. 7(277), 277ra230 (2015). https://doi.org/10.1126/scitranslmed.aaa12 60

20. S. Gao, H. Lin, H. Zhang, H. Yao, Y. Chen, J. Shi, Nanocatalytic tumor therapy by biomimetic dual inorganic nanozymecatalyzed cascade reaction. Adv. Sci. 6(3), 1801733 (2019). https://doi.org/10.1002/advs.201801733

21. J. Wang, Y. Zhang, E. Archibong, F.S. Ligler, Z. Gu, Leveraging $\mathrm{H}_{2} \mathrm{O}_{2}$ levels for biomedical applications. Adv. Biosyst. 1(9), 1700084 (2017). https://doi.org/10.1002/adbi.201700084

22. D. Deng, C. Hao, S. Sen, C. Xu, P. Král, N.A. Kotov, Template-free hierarchical self-assembly of iron diselenide nanoparticles into mesoscale hedgehogs. J. Am. Chem. Soc. 139(46), 16630-16639 (2017). https://doi.org/10.1021/ jacs.7b07838

23. D. Deng, Y. Chen, J. Cao, J. Tian, Z. Qian, S. Achilefu, Y. Gu, High-quality $\mathrm{CuInS}_{2} / \mathrm{ZnS}$ quantum dots for in vitro and in vivo bioimaging. Chem. Mater. 24(15), 3029-3037 (2012). https:// doi.org/10.1021/cm3015594

24. C. Cheng, S. Li, A. Thomas, N.A. Kotov, R. Haag, Functional graphene nanomaterials based architectures: biointeractions, fabrications, and emerging biological applications. Chem. Rev. 117(3), 1826-1914 (2017). https://doi.org/10.1021/acs.chemr ev.6b00520

25. P.N. Manghnani, W. Wu, S. Xu, F. Hu, C. Teh, B. Liu, Visualizing photodynamic therapy in transgenic zebrafish using organic nanoparticles with aggregation-induced emission. Nano-Micro Lett. 10(4), 61 (2018). https://doi.org/10.1007/ s40820-018-0214-4

26. C.R. Gordijo, A.Z. Abbasi, M.A. Amini, H.Y. Lip, A. Maeda et al., Design of hybrid $\mathrm{MnO}_{2}$-polymer-lipid nanoparticles with tunable oxygen generation rates and tumor accumulation for cancer treatment. Adv. Funct. Mater. 25(12), 1858-1872 (2015). https://doi.org/10.1002/adfm.201404511

27. Y. Yang, L. Ma, C. Cheng, Y. Deng, J. Huang et al., Nonchemotherapic and robust dual-responsive nanoagents with ondemand bacterial trapping, ablation, and release for efficient wound disinfection. Adv. Funct. Mater. 28(21), 1705708 (2018). https://doi.org/10.1002/adfm.201705708

28. S. Shi, F. Chen, S. Goel, S.A. Graves, H. Luo et al., In vivo tumor-targeted dual-modality PET/optical imaging with a yolk/shell-structured silica nanosystem. Nano-Micro Lett. 10(4), 65 (2018). https://doi.org/10.1007/s40820-018-0216-2

29. G. Yang, L. Xu, Y. Chao, J. Xu, X. Sun, Y. Wu, R. Peng, Z. Liu, Hollow $\mathrm{MnO}_{2}$ as a tumor-microenvironment-responsive biodegradable nano-platform for combination therapy favoring antitumor immune responses. Nat. Commun. 8(1), 902 (2017). https://doi.org/10.1038/s41467-017-01050-0

30. M. Song, T. Liu, C. Shi, X. Zhang, X. Chen, Bioconjugated manganese dioxide nanoparticles enhance chemotherapy response by priming tumor-associated macrophages toward M1-like phenotype and attenuating tumor hypoxia. ACS Nano 10(1), 633-647 (2016). https://doi.org/10.1021/acsnano.5b067 79 
31. R.-Q. Li, C. Zhang, B.-R. Xie, W.-Y. Yu, W.-X. Qiu, H. Cheng, $\mathrm{X}$.-Z. Zhang, A two-photon excited $\mathrm{O}_{2}$-evolving nanocomposite for efficient photodynamic therapy against hypoxic tumor. Biomaterials 194, 84-93 (2019). https://doi.org/10.1016/j. biomaterials.2018.12.017

32. L. Meng, Y. Cheng, X. Tong, S. Gan, Y. Ding et al., Tumor oxygenation and hypoxia inducible factor-1 functional inhibition via a reactive oxygen species responsive nanoplatform for enhancing radiation therapy and abscopal effects. ACS Nano 12(8), 8308-8322 (2018). https://doi.org/10.1021/acsna no. 8 b03590

33. Y. Zhang, F. Wang, C. Liu, Z. Wang, L. Kang et al., Nanozyme decorated metal-organic frameworks for enhanced photodynamic therapy. ACS Nano 12(1), 651-661 (2018). https://doi. org/10.1021/acsnano.7b07746

34. Z. Wang, Y. Zhang, E. Ju, Z. Liu, F. Cao, Z. Chen, J. Ren, X. $\mathrm{Qu}$, Biomimetic nanoflowers by self-assembly of nanozymes to induce intracellular oxidative damage against hypoxic tumors. Nat. Commun. 9(1), 3334 (2018). https://doi. org/10.1038/s41467-018-05798-x

35. C. Yao, W. Wang, P. Wang, M. Zhao, X. Li, F. Zhang, Nearinfrared upconversion mesoporous cerium oxide hollow biophotocatalyst for concurrent $\mathrm{pH}-/ \mathrm{H}_{2} \mathrm{O}_{2}$-responsive $\mathrm{O}_{2}$-evolving synergetic cancer therapy. Adv. Mater. 30(7), 1704833 (2018). https://doi.org/10.1002/adma.201704833

36. T. Finkel, M. Serrano, M.A. Blasco, The common biology of cancer and ageing. Nature 448, 767-774 (2007). https://doi. org/10.1038/nature05985

37. X. Song, J. Xu, C. Liang, Y. Chao, Q. Jin, C. Wang, M. Chen, Z. Liu, Self-supplied tumor oxygenation through separated liposomal delivery of $\mathrm{H}_{2} \mathrm{O}_{2}$ and catalase for enhanced radioimmunotherapy of cancer. Nano Lett. 18(10), 6360-6368 (2018). https://doi.org/10.1021/acs.nanolett.8b02720

38. M. Thiel, C.C. Caldwell, S. Kreth, S. Kuboki, P. Chen et al., Targeted deletion of HIF- $1 \alpha$ gene in T cells prevents their inhibition in hypoxic inflamed tissues and improves septic mice survival. PLoS ONE 2(9), e853 (2007). https://doi. org/10.1371/journal.pone.0000853

39. W.-P. Li, C.-H. Su, Y.-C. Chang, Y.-J. Lin, C.-S. Yeh, Ultrasound-induced reactive oxygen species mediated therapy and imaging using a fenton reaction activable polymersome. ACS Nano 10(2), 2017-2027 (2016). https://doi.org/10.1021/acsna no. 5 b06175

40. J. Wang, D. Xu, T. Deng, Y. Li, L. Xue, T. Yan, D. Huang, D. Deng, Self-decomposable mesoporous doxorubicin@ silica nanocomposites for nuclear targeted chemo-photodynamic combination therapy. ACS Appl. Nano Mater. 1(4), 19761984 (2018). https://doi.org/10.1021/acsanm.8b00486

41. S. Zhang, Z. Chu, C. Yin, C. Zhang, G. Lin, Q. Li, Controllable drug release and simultaneously carrier decomposition of $\mathrm{SiO}_{2}$-drug composite nanoparticles. J. Am. Chem. Soc. 135(15), 5709-5716 (2013). https://doi.org/10.1021/ja312 3015

42. S.B. Lee, J.-E. Lee, S.J. Cho, J. Chin, S.K. Kim et al., Crushed gold shell nanoparticles labeled with radioactive iodine as a theranostic nanoplatform for macrophage-mediated photothermal therapy. Nano-Micro Lett. 11, 36 (2019). https ://doi.org/10.1007/s40820-019-0266-0

43. H. Chen, J. Tian, W. He, Z. Guo, $\mathrm{H}_{2} \mathrm{O}_{2}$-activatable and $\mathrm{O}_{2}$-evolving nanoparticles for highly efficient and selective photodynamic therapy against hypoxic tumor cells. J. Am. Chem. Soc. 137(4), 1539-1547 (2015). https://doi. org/10.1021/ja511420n

44. K. Synnestvedt, G.T. Furuta, K.M. Comerford, N. Louis, J. Karhausen et al., Ecto-5'-nucleotidase (CD73) regulation by hypoxia-inducible factor-1 mediates permeability changes in intestinal epithelia. J. Clin. Investig. 110(7), 993-1002 (2002). https://doi.org/10.1172/jci0215337

45. H.K. Eltzschig, D. Kohler, T. Eckle, T. Kong, S.C. Robson, S.P. Colgan, Central role of sp1-regulated CD39 in hypoxia/ ischemia protection. Blood 113(1), 224-232 (2009). https:// doi.org/10.1182/blood-2008-06-165746

46. A. Palazon, A.W. Goldrath, V. Nizet, R.S. Johnson, HIF transcription factors, inflammation, and immunity. Immunity 41(4), 518-528 (2014). https://doi.org/10.1016/j.immun i.2014.09.008

47. K.M. Mahoney, P.D. Rennert, G.J. Freeman, Combination cancer immunotherapy and new immunomodulatory targets. Nat. Rev. Drug Discov. 14, 561 (2015). https://doi. org/10.1038/nrd4591

48. G.L. Klement, Eco-evolution of cancer resistance. Sci. Transl. Med. 8(327), 327 fs325 (2016). https://doi. org/10.1126/scitranslmed.aaf3802

49. P.M. Enriquez-Navas, Y. Kam, T. Das, S. Hassan, A. Silva, P. Foroutan et al., Exploiting evolutionary principles to prolong tumor control in preclinical models of breast cancer. Sci. Transl. Med. 8(327), 327ra324 (2016). https://doi. org/10.1126/scitranslmed.aad7842

50. D.-W. Zheng, J.-L. Chen, J.-Y. Zhu, L. Rong, B. Li et al., Highly integrated nano-platform for breaking the barrier between chemotherapy and immunotherapy. Nano Lett. 16(7), 4341-4347 (2016). https://doi.org/10.1021/acs.nanol ett.6b01432

51. W. Chen, Y. Wang, M. Qin, X. Zhang, Z. Zhang, X. Sun, Z. $\mathrm{Gu}$, Bacteria-driven hypoxia targeting for combined biotherapy and photothermal therapy. ACS Nano 12(6), 5995-6005 (2018). https://doi.org/10.1021/acsnano.8b02235

52. Y.N. Zhao, X.X. Sun, G.N. Zhang, B.G. Trewyn, I.I. Slowing, V.S.-Y. Lin, Interaction of mesoporous silica nanoparticles with human red blood cell membranes: size and surface effects. ACS Nano 5(2), 1366-1375 (2011). https://doi. org/10.1021/nn103077k

53. T. Maj, W. Wang, J. Crespo, H. Zhang, W. Wang et al., Oxidative stress controls regulatory $\mathrm{T}$ cell apoptosis and suppressor activity and PD-L1-blockade resistance in tumor. Nat. Immunol. 18, 1332 (2017). https://doi.org/10.1038/ ni. 3868

54. C. Wang, Y. Ye, Q. Hu, A. Bellotti, Z. Gu, Tailoring biomaterials for cancer immunotherapy: emerging trends and future outlook. Adv. Mater. 29(29), 1606036 (2017). https://doi. org/10.1002/adma.201606036 
55. A. Facciabene, X. Peng, I.S. Hagemann, K. Balint, A. Barchetti et al., Tumour hypoxia promotes tolerance and angiogenesis via ccl28 and Treg cells. Nature 475, 226 (2011). https ://doi.org/10.1038/nature10169

56. N. Casares, M.O. Pequignot, A. Tesniere, F. Ghiringhelli, S. Roux et al., Caspase-dependent immunogenicity of doxorubicin-induced tumor cell death. J. Exp. Med. 202(12), 16911701 (2005). https://doi.org/10.1084/jem.20050915
57. D. Hannani, A. Sistigu, O. Kepp, L. Galluzzi, G. Kroemer, L. Zitvogel, Prerequisites for the antitumor vaccine-like effect of chemotherapy and radiotherapy. Cancer J. 17(5), 351-358 (2011). https://doi.org/10.1097/PPO.0b013e3182325d4d

58. S.L. Topalian, C.G. Drake, D.M. Pardoll, Immune checkpoint blockade: a common denominator approach to cancer therapy. Cancer Cell 27(4), 450-461 (2015). https://doi.org/10.1016/j. ccell.2015.03.001 